\title{
Cooperative Intersection Support System Based on Mirroring Mechanisms Enacted by Bio-Inspired Layered Control Architecture
}

\author{
Mauro Da Lio, Member, IEEE, Alessandro Mazzalai, and Marco Darin
}

\begin{abstract}
This paper presents a cooperative intersection support system implemented with an artificial cognitive system enacted by an agent that replicates human driver longitudinal sensorimotor control in the application domain. The engineering of the agent exploits recent ideas from Cognitive Science, among which the notion of mirroring (the agent discovering driver intentions by simulating possible human actions). The paper introduces the design principles for the agent and the following implementation. The system is evaluated with experimental data and compared to state of the art implementations that use different approaches.
\end{abstract}

Index Terms-Advanced driver assistance systems, intersection support, driver modeling, layered control architectures, artificial cognitive systems.

\section{INTRODUCTION}

$\mathbf{T}$ HIS paper presents a cooperative intersection support system, warning the driver for collision and red/yellow traffic light crossing, that uses bio-inspired layered control architecture to 'mirror' the human driver.

'Mirroring' here refers to Cognitive Science [1]-[7]; namely to using an external agent -the artificial system- to figure out the actions that can be human-directed in the given context and, within these, to find which goal best explains the human behavior observed so far.

This active way of discovering human intentions is termed 'generative' in the robotics community, as opposed to approaches based on behavioral pattern classification [8].

By fitting the observed behavior onto context-related generated affordances ${ }^{1}$ generative approaches interpret human behavior as the manifestation of goal-directed actions [10]. This often provides the means to resolve ambiguities that would be difficult to disambiguate otherwise (the same motor pattern may have different meanings in different contexts).

Manuscript received February 7, 2017; revised May 28, 2017; accepted July 16, 2017. This work was supported in part by the local province of Trento (PAT), Italy, under Grant e2Call and in part by the European Commission under Grant FP7 246587 (interactIVe.). The Associate Editor for this paper was N. Geroliminis. (Corresponding author: Mauro Da Lio.)

M. Da Lio and A. Mazzalai are with the Department of Industrial Engineering, University of Trento, 38123 Povo, Italy (e-mail: mauro.dalio@unitn.it; alessandro.mazzalai@unitn.it).

M. Darin is with the CRF, the Research Center of FCA (Fiat Chrysler Automobiles), 38123 Povo, Italy (e-mail: marco.darin@crf.it).

Color versions of one or more of the figures in this paper are available online at http://ieeexplore.iee.org.

Digital Object Identifier 10.1109/TITS.2017.2731901

${ }^{1}$ The notion of affordance has been introduced by Gibson [9] to indicate all actions, latent in the current environment, that an agent is capable of.
Application to driver assistance system of the above idea was introduced in general terms in [11]. In this paper, we develop, and validate with experiments, an agent for the scenario of cooperative intersections warning.

Beyond the application itself, the main contribution of this paper concerns also the methodology for building Driver Support Systems by using a conceptual framework that is widely accepted in human natural cognition.

\section{A. Aimed Application}

The system here described was conceived, in the funding project 'e2Call', to work with as little as: V2X communication, global positioning system (e.g., the GPS), and digital maps. With the forthcoming introduction of emergency call $\left(\mathrm{e}-\right.$ call $\left.^{2}\right)$ systems as standard equipment -which, in the EU, will be mandatory in 2018-, the former two conditions will be satisfied by every new vehicle; the latter (digital maps) could be provided locally by infrastructure communication, if not already available in the vehicle. Hence, the main aim of the e2Call project was to study preventive safety intersection support function for possibly large-scale adoption.

The system is simple enough (in particular because it does not need sensors) that it may be extended easily to motorcycles, thus addressing this particular category of vulnerable road users, which are at higher risk and for which less solutions are prospectively available.

\section{B. Organization of the Work}

The paper is organized as follows. Section II describes the related state of the art. Section III introduces the application use cases. Section IV motivates the chosen system architecture. Section V gives the implementation and section VI the system evaluation and experiments.

\section{RELATED WORK}

Intersections are among the main loci of collision and various measures have been studied for their safety. Some form of vehicle and infrastructure cooperation is almost always assumed. This may be as little as vehicles communicating each other position and velocity (cooperation at the perception level) or involve negotiation, cooperative planning, scheduling,

\footnotetext{
${ }^{2}$ Future e-call systems could rely on $4 \mathrm{G} / 5 \mathrm{G}$ connectivity and positioning systems with decimeter/centimeter scale accuracy.
} 
vehicle and infrastructure control, automated driving coordination, etc. (cooperation at the control level). Concerning the latter, three recent reviews are [12]-[14]. For example [15] describes an infrastructure-based system that optimizes the trajectories of Cooperative ACC equipped vehicles. A similar work is [16], which however describes a decentralized algorithm. Conversely, [17] deals with negotiating and scheduling the traffic light phases, while [18] deals with cooperative driving scheduling in un-signalized intersections. For systems that aim at cooperative control, various optimization approaches may be used, and the goals are typically to improve traffic throughput, minimize delays, improve energy efficiency and comfort while ensuring safety.

A different approach to intersection safety is based on the concept of Cooperative Collision Warning [19], which are potentially inexpensive -and hence potentially widespreadsystems that act by warning the driver in case of human errors. The responsibility of driving is left to the driver (automation level 0) but the system may prevent error, and works, albeit with reduced performance, even in mixed traffic where equipped and non-equipped vehicles co-exist. These systems might be engineered even without communications, but of course they take great advantage of V2X technologies that provide information for objects occluded to the line of sight (and may even allow to save expensive range sensors). Given the aims stated in Section I.A this paper falls in this category.

A cooperative intersection collision warning system based on roadside sensors is proposed in [20] with emphasis on the Korean road networks. The system aims at using roadside sensors, communications and existing onboard units (similar to this work) to provide collision warnings. The work uses Time To Collision (TTC) as an indicator of collision risk.

In the United States, the Cooperative Intersection Collision Avoidance Systems (CICAS) project studied collision support systems for intersection scenarios, and in particular for violation at stop and traffic lights and to support left turns [21]. Concerning the algorithms underlying the generation of warnings, a paper by Aoude et al. [22] discusses various approaches to classify the incoming vehicles as possible violators. The simplest traditional methods rely on the use of surrogate indicators, such as Time to Intersection (TTI), Required Deceleration Policy (RDP) and Speed-Distance Regression models (SDR). More advanced approaches are based on Hidden Markov Models (HMM) and Support Vector Machines with Bayesian Filtering (SVM-BF). The latter require observing the speed and distance of an approaching vehicle till it is quite close to the intersection (typically 1.6 or 2 seconds) and then classify the observed time series into the categories of stopping and violator vehicles. The classifier is trained with a very large naturalistic driving data set.

Similar classification approaches may be found in other works; notably [23], which extends the application domain to the classification among four possible intentions (no action, stop, creep and go) in yield scenarios without priority. One important finding was that prediction of the vehicle with priority was necessary and, consequently, observations of the second vehicle were included in the training of the three tested classifiers (SVM, Random Forest and k-nearest neighbors).

In the European Union, the INTERSAFE-2 project studied Cooperative Intersection Safety System for both warning and intervention strategies [24]. Concerning the algorithms, the project implements simple (linear) trajectory forecasting of objects, which, to account for the stochastic nature of trajectories, are modeled with an occupied circular region of increasing radius. When the regions representing two objects overlap, a risk of collision is detected. The time at which this event occur (Time to Collision, TTC) is compared to standard reaction time (2s) to assess the risk level: the risk is set to 1 for TTC $<2 \mathrm{~s}$ and decreases linearly to zero for TTC $=5 \mathrm{~s}$ [25].

The EU project SAFERIDER, focused on producing assistance systems for motorcycle riders, also studied collision support for these particular road users. The project used nonlinear optimal control to generate humanlike trajectories for intersection negotiation. These are used as gold-standard ('reference maneuver') to compare with the actual behavior of the human driver (hence the notion of mirroring is somewhat implicit). The amount of correction that would be needed to steer the current motorcycle state (as driven so far by the human driver) into the safe final state (as assessed by the reference maneuver) was used to assess the risk level. If the amount of steering rate and acceleration rate required to produce the safe reference maneuver exceeded a given threshold, warnings were issued (two thresholds had been chosen for advisory and cautionary warnings) [26], [27].

\section{Application Scenario}

The application scenario is illustrated schematically in Fig.1, left. We identify two use cases: 1) stop/traffic light, 2) intersecting trajectories.

1) Use case 1 concerns the compliance with traffic lights or with mandatory stop lines. The vehicle must stop at location $s$, near the stop line (or earlier than $s$ ), if the traffic light signalizes to stop or if there is a mandatory stop.

2) Use case 2 deals with intersecting lanes. In this case point $c$ labels the intersection of the centerlines and points $s$ and $l$ indicate where vehicles enter and clear the intersection area. We can identify two sub-cases: 1) the vehicles leave the intersection in distinct roads and 2) the vehicles leave the intersection on the same road (and hence share the same lane after point $s$ ).

\section{System ARChitecture}

The main goal of this paper is to engineer a system that is able to work like a model of the human sensorimotor system, so that it can be used to mirror the human driver [6]. For this, the architecture of human motor control is considered as a useful engineering guideline, as follows.

\section{A. Layered Control Architecture}

Layered control architectures are widely acknowledged for modelling hierarchical sensorimotor adaptive behavior [28], 

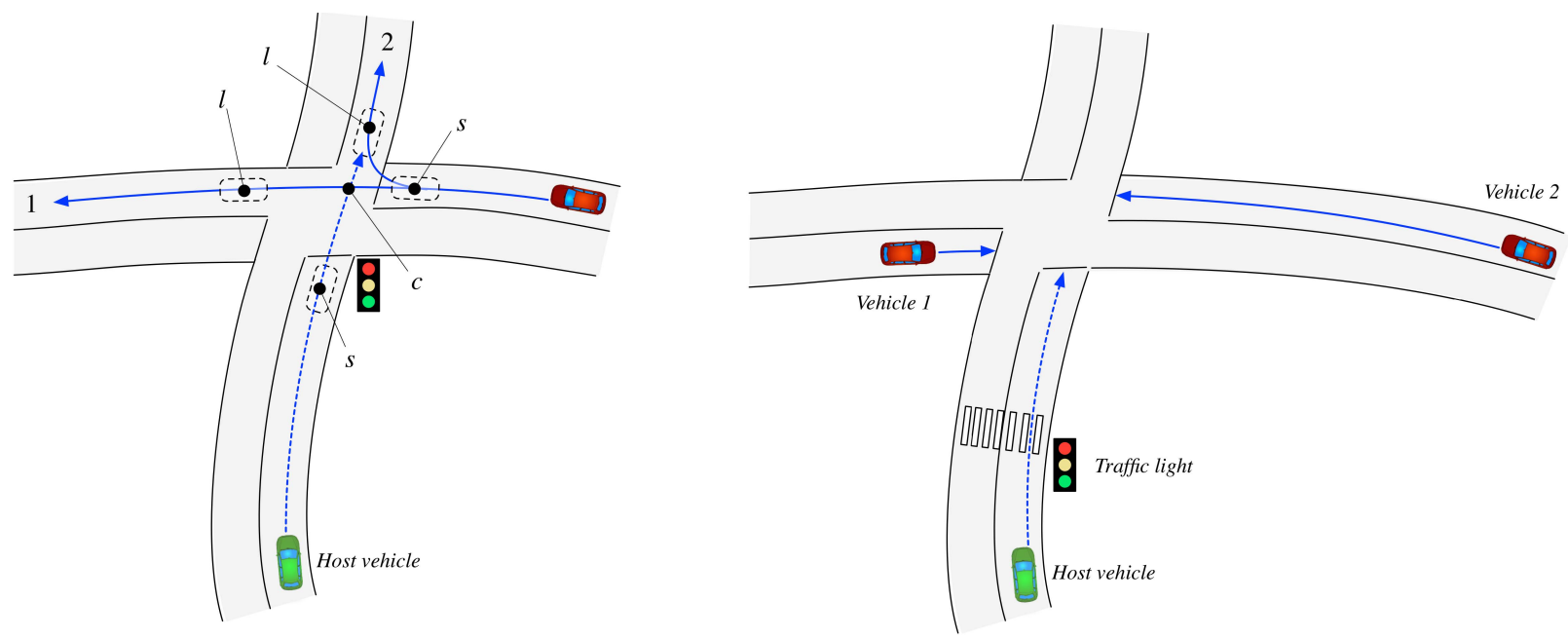

Fig. 1. Intersection scenarios. Left: vehicles enter the intersection area after trespassing point $s$ (the last possible stop point) and leave the area at point $l$. The point where the trajectories intersects is labelled as $c$. Vehicles may leave the intersection on different roads (path 1) or on the same road (path 2). Intersections may be either signalized by traffic lights or not. Right: one example of a situation dealing with several simultaneous conditions: a traffic light regulates a pedestrian crossing and two vehicles are on the host vehicle path, at different distance to the intersection, on an un-signalized intersection.

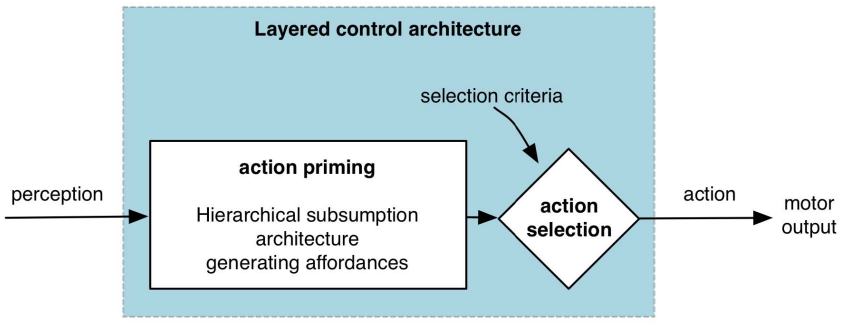

Fig. 2. The system adopts layered control architecture with two main building blocks: 1) action priming, which generates all possible humanlike actions in the given situation and 2) action selection, which selects the action matching the actual human behavior.

in particular of humans. They are similar to subsumption architectures -well known in robotics [29]- from which they depart mainly for the use of a centralized action-selection mechanism [28], which provides consistent and efficient conflict resolution mechanisms.

Hence, for engineering an agent with humanlike sensorimotor behaviors, adopting a layered control architecture may be a very good choice.

Following [11], the artificial agent of this paper is called 'co-driver.' Fig.2 gives a high-level functional decomposition of the co-driver. It is made of two distinct building blocks, which implement the two distinct moments of 1) generation of affordances (action priming) and 2) mirroring (action selection).

We might look at Fig.2 as a process where hypotheses about possible driver goals are made (action priming) and tested against actual driver behavior (action selection). However, we like looking at Fig.2 as a digital implementation of the high-level sensorimotor loop postulated by the 'affordance competition hypothesis' [30] for humans. According to this, action priming occurs in the cortex dorsal stream -where multiple affordances are instantiated simultaneously- and action selection occurs in the basal ganglia, following high-level directives (selection criteria) specified by various areas of the brain ([30], Fig.1).

Implicit in this scheme is the idea that 'mirroring' is a particular type of action selection where the selected affordance is the most similar to the observed human behavior (e.g., [11], Fig.7).

However, it may be worth noting that for the application of this paper-which is preventive safety-, we do not even need to discover the human intention exactly: we only need to assess whether there exists at least one affordance within the human driver reach (section V.B).

\section{B. Mirroring for Advisory and Cautionary Warning Levels}

Driver assistance systems generally produce two levels of warning. Advisory warnings are produced when the driver behavior is incorrect but there is no imminent danger. Cautionary warnings are produced in case of imminent danger and mean that some corrective action is immediately required.

To implement the two levels, the human driver is mirrored twice.

Firstly, for advisory level, the parameters of the sensorimotor architecture (Fig.1) are set to values that represent a correct driver (e.g., crossing traffic light rigorously with the green phase, leaving correct time gaps in an intersection with another vehicle, etc.).

Secondly, for cautionary level, the parameters of the co-driver sensorimotor architecture are somewhat relaxed (e.g., crossing the traffic light in the grace time of the yellow phase, leaving tighter time gaps at intersection with another vehicle, etc.).

Hence the human driver is compared with both a correct and a slightly incorrect co-driver. If the human behavior fits the correct co-driver, then no warning is issued. If it fits the slightly incorrect driver, then an advisory warning is issued (e.g., meaning 'you are going to cross the traffic light with 
the yellow phase'). If the last is not fitted, then a cautionary warning is issued (e.g., meaning 'you will cross in the red phase').

\section{CO-DRIVER IMPLEMENTATION}

\section{A. Action Priming}

In layered/subsumption conceptual frameworks, complex actions can be seen as hierarchies of simpler and simpler behaviors. Atomic motor units -called motor primitives- form the bottommost layer of the hierarchy. This holds both for biological systems [31], [32] and for robots [29], [33].

In our application, for building both traffic light and intersection behaviors, we need only a shallow hierarchy and only two primitives, for either a) stopping or b) passing a given position within a given time interval and speed interval.

For the construction of the motor primitives -so that they resemble human motor primitives (which, as said, is required for mirroring)- one may refer to the existing literature on optimality principles of human sensorimotor control [34]-[42], and in particular to the principle of reducing neural motor noise effects [39]-[42], which is well approximated by minimum square jerk criterion (albeit there is no consensus on the exact formulation).

Appendix 1 derives the motor primitives with the minimum square jerk criterion. They happen to be $5^{\text {th }}$ order polynomial, i.e., $s(t)=c_{1} t+\frac{1}{2} c_{2} t^{2}+\frac{1}{6} c_{3} t^{3}+\frac{1}{24} c_{4} t^{4}+\frac{1}{120} c_{5} t^{5}$, where $s(t)$ represents the travelled distance as a function of time (A1.2).

Action priming is thus, in practice, the computation of the polynomials for each goal of each uses case, as detailed in the following.

1) Traffic Light: A driver approaching a traffic light may have two possible goals:

a) Stopping at the traffic light or earlier (point ' $s$ ' in Fig.1, left).

b) Trespassing the traffic light within a given time interval and within a given speed interval.

The above alternatives are shown with an example in Fig.3:

a) motor primitives leading to stop may be represented either by polynomial $m_{1}$ or by any primitive slower than $m_{1}$ (the green shaded area below $m_{1}$ );

$b$ ) motor primitives trespassing the traffic light may be $m_{2}$ or $m_{3}$, or any primitive between $m_{2}$ and $m_{3}$ (the light blue shaded area between $m_{2}$ and $m_{3}$ ).

To represent the set of possible actions we need to compute the extremal primitives $m_{1}, m_{2}$ and $m_{3}$.

Following appendix 1 , we concisely write (where $a_{0}, v_{0}$ are the current acceleration and velocity of the host vehicle):

$$
\begin{aligned}
m_{1} & =\operatorname{Stop}\left(a_{0}, v_{0}, x_{s}\right) \\
\left\{m_{2}, m_{3}\right\} & =\operatorname{Pass}\left(a_{0}, v_{0}, x_{s}, T_{1}, T_{2}, v_{\min }, v_{\max }\right)
\end{aligned}
$$

The first equation in (1) returns the primitive (the polynomial) stopping at the traffic light position $x_{s}$ according to Appendix 1.A.

The second equation in (1) generates the slowest and fastest primitives that cross point $x_{s}$ at time $T \in\left[T_{1}, T_{2}\right]$, and with velocity $v_{s} \in\left[v_{\min }, v_{\max }\right]$, according to appendix 1.B.

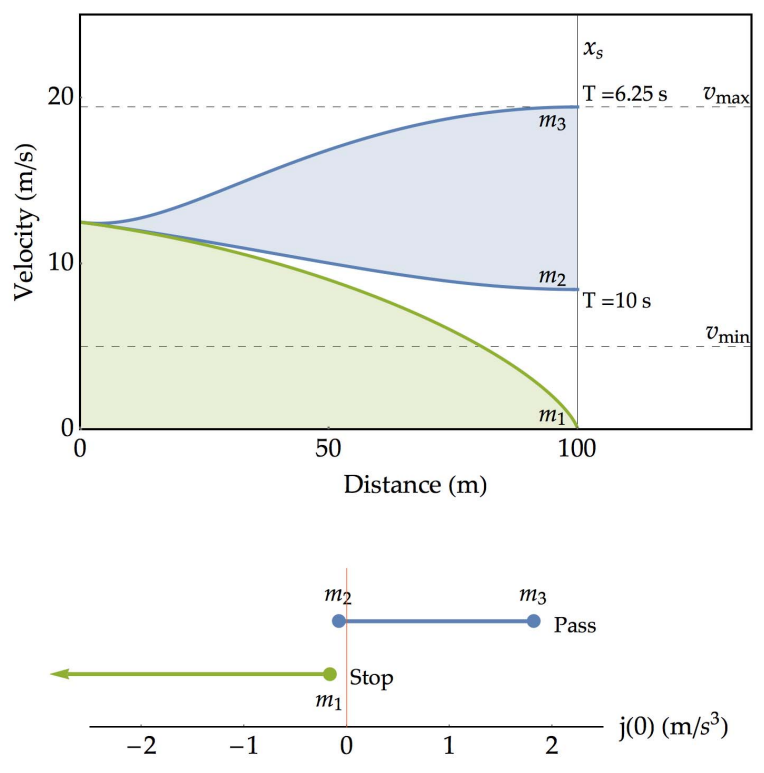

Fig. 3. Top. Set of actions that are possible at a traffic light. In this example, the initial speed of the vehicle is $v_{0}=45 \mathrm{~km} / \mathrm{h}$, the initial acceleration is $a_{0}=-0.5 \mathrm{~m} / \mathrm{s}^{2}$, and the traffic light is at $x_{s}=100 \mathrm{~m}$ ahead. The host vehicle can either stop at $x_{s}$ or earlier (which is represented by the shaded area under $m_{1}$ ) or can pass the traffic light (which is represented by the shaded area between $m_{2}$ and $m_{3}$ ). The latter is allowed only within the speed range $v_{\text {min }}-v_{\max }$ and the time range $T_{1}-T_{2}$. In this example $T_{1}=0$ and $T_{2}=10 \mathrm{~s}$ (the traffic light is currently in the green phase and will stay so for $10 \mathrm{~s}$ ). Both intervals $v_{\min }-v_{\max }$ and $T_{1}-T_{2}$ may limit the transit: in this example, the fastest maneuver $\left(m_{3}\right)$ is limited by $v_{\max }$ and the slowest maneuver is limited by $T_{2}$ (driving slower than $m_{2}$ will reach the traffic light after $T_{2}$ ). Bottom. Projection of actions in one-dimensional space corresponding to the longitudinal control (jerk) at time $t=0$. In the example, trespassing the traffic light requires longitudinal jerk spanning the interval from slightly less than 0 (for trespassing at $T=10 \mathrm{~s}$ ) to nearly $2 \mathrm{~m} / \mathrm{s}^{3}$ (to accelerate for trespassing at $T=6.25 \mathrm{~s}$ ). This is represented as the 'Pass' set in the chart. To stop, the longitudinal required control would be slightly less than 0 and stopping earlier would require further negative jerk (hence the 'Stop' set shown in the chart extends to $-\infty$, represented by the leftward pointing arrow).

The interval $\left[T_{1}, T_{2}\right]$ represents the time slot available for crossing. This information must be communicated by the infrastructure. We set $T_{1}$ at the time the traffic light phase will become green (albeit one might prefer setting $T_{1}$ slightly later to allow some margin). For advisory warning, we set $T_{2}$ at the time the traffic light will turn to the yellow phase. For cautionary warning, we set $T_{2}$ at the yellow phase onset plus the grace time (typically 2-3 s after the yellow onset).

The interval $\left[v_{\min }, v_{\max }\right]$ prevents the vehicle crossing the intersection too fast (e.g., $v_{\max }$ above the speed limit) or too slow. In particular, the minimum speed $v_{\min }$ aims at guaranteeing that the vehicle clears the intersection in a reasonable time (alternatively and better, one may compute a second set of motor primitives for the exit point $x_{l}$, to ensure that the exit is also trespassed within the $\left[T_{1}, T_{2}\right]$ time slot).

2) Intersection Motor Primitive: For negotiating an intersection with another vehicle there are two affordances similar to the traffic light case:

c) Stopping at the stop line or earlier (stopping at point ' $s$ ' or earlier in Fig.1).

d) Crossing the intersection (point ' $c$ ' in Fig.1) within a given speed interval and while leaving a safe time gap with the other vehicle. 


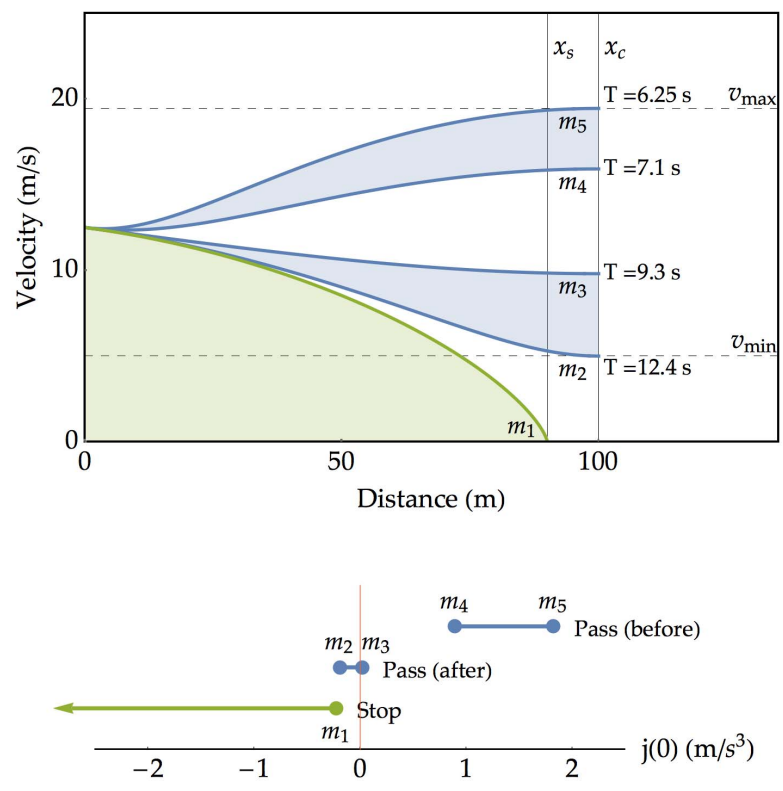

Fig. 4. Top. Set of actions that are possible for the intersection with another vehicle (Fig.1, left). In this example, the initial speed of the host vehicle is $v_{0}=45 \mathrm{~km} / \mathrm{h}$ and the initial acceleration is $a_{0}=-0.5 \mathrm{~m} / \mathrm{s}^{2}$ (same as for Fig.3). The stop line is at $x_{s}=90 \mathrm{~m}$ ahead. The host vehicle can either stop at $x_{s}$ or earlier (which is represented by the green shaded area under $m_{1}$ ) or can cross the intersection. If it crosses the intersection, it can either pass before or after the opponent vehicle, which are the two light-blue shaded areas between $m_{3}-m_{2}$ or $m_{5}-m_{4}$. The time slot $T_{1}-T_{2}$ (in this example $T_{1}=7.1$ and $T_{2}=9.3 \mathrm{~s}$ for sake of illustration) is reserved for the opponent vehicle (3). Bottom. Projection of actions in the $j(0)$ subspace. In the example, crossing is possible either before or after the opponent vehicle.

The case $c$ is the same of $a$ for the traffic light (stopping at ' $s$ '). The case $d$ is 'opposite' to $b$, in the sense that the host vehicle must not use the intersection in the time slot $\left[T_{1}, T_{2}\right]$, which is reserved for the intersecting vehicle; i.e., the host vehicle can either pass after $T_{2}\left(T \in\left[T_{2}, \infty\right]\right)$ or before $T_{1}$ $\left(T \in\left[0, T_{1}\right]\right)$. The set of admissible maneuvers is thus made of three subsets:

$$
\begin{aligned}
m_{1} & =\operatorname{Stop}\left(a_{0}, v_{0}, x_{s}\right) \\
\left\{m_{2}, m_{3}\right\} & =\operatorname{Pass}\left(a_{0}, v_{0}, x_{c}, T_{2}, \infty, v_{\min }, v_{\max }\right) \\
\left\{m_{2}, m_{3}\right\} & =\operatorname{Pass}\left(a_{0}, v_{0}, x_{c}, 0, T_{1}, v_{\min }, v_{\max }\right)
\end{aligned}
$$

Fig.4 gives an example where all the three subsets are present. Note however that a 'Pass' subset may be empty (if both are missing the only option remaining is to stop). Note also that the Pass primitives in (2) are computed on the intersection point ' $c$ ', whereas they were computed on ' $s$ ' for the traffic light.

For the computation of the inhibited time slot $\left[T_{1}, T_{2}\right]$ the trajectory of the intersecting vehicle has to be predicted. There are two options: a) the intentions of the other vehicle (e.g., as assessed by a system onboard that vehicle) are communicated; b) the intentions of the other vehicle are estimated with a mirroring process.

While option a) may look appealing, we implemented, for the moment, option b), because it does not need the communication of motor primitives (which would require extensions of the communication protocols). In fact, intentions can be estimated with speed, position and acceleration of the other vehicle, which are likely to be available even if the other vehicle is not equipped with the system. Note that $b$ ) is actually the process that human drivers use.

For this, we have implemented a simple mirroring process ${ }^{3}$ which, given the velocity and acceleration of the intersecting vehicle, produces an estimated motor primitive and then computes the times the vehicle enters $T_{i n}$ and leaves the intersection $T_{\text {out }}$ (respectively reaches points $s$ and $l$ ).

Hence, given $T_{g}$-the gap a human driver accepts for intersecting trajectories- the inhibited time slot is defined as:

$$
\begin{aligned}
& T_{1}=T_{\text {in }}-T_{g} \\
& T_{2}=T_{\text {out }}-T_{g}
\end{aligned}
$$

While $T_{\text {in }}$ and $T_{\text {out }}$ are stochastic in nature, their variability, even with the simplified mirroring mechanism above, is smaller than the usual values for $T_{g}$, when the vehicles are near the intersection (one might argue that humans adopt the time gap $T_{g}$ for the very purpose of accounting for uncertainties in $T_{\text {in }}$ and $T_{\text {out }}$ and hence ensuring that the intersection is clear before $T_{1}$ or after $T_{2}$ ).

Concerning the accepted gap there are many studies in the literature, and one recent review is [43].

In the implementation tested in the experiments below, we initially assumed $T_{g}=4 \mathrm{~s}$ for advisory warning and $T_{g}=3 \mathrm{~s}$ for cautionary warning, based on the literature values [43]. These assumptions were confirmed during the test campaign.

However, the literature shows that these values may vary depending on the actual geometry and the priority of the roads, for through and right turns, for different vehicle types, whether the traffic is heterogeneous, etc. Hence, modelling the critical time gap in different situations and geometry may be a valuable improvement.

\section{B. Action Selection}

Fig.1, left, showed a relatively simple situation with only two 'objects': one traffic light and one opponent vehicle. Correspondingly, Fig. 3 showed the actions that are compatible with the traffic light and Fig.4 those that are possible with the opponent obstacle.

If there were only one 'object', e.g., say one opponent vehicle, guessing the intentions of the host driver could be based on that object alone. In the example of Fig.4 one might guess that, since crossing is possible (and we assume that a driver wishes to cross whenever possible), the driver will almost surely cross the intersection, and, because he/she is decelerating, he/she will likely opt for crossing after the opponent vehicle, i.e., choosing one behavior between $m_{2}$ and $m_{3}$ (in the range $T=9.3-12.4 s$, Fig.4).

However, real situations may involve the presence of several simultaneous threats such as, e.g., Fig.1 right, which

\footnotetext{
${ }^{3}$ For mirroring, one can make the hypotheses that the intersecting vehicle is either going to stop or pass, such as in Fig.3, but with relaxed $T_{1}, T_{2}, v_{\min }$, $v_{\max }$ to account for possible incorrect behaviors. Hence, the most likely motor primitive, which corresponds to $j(0)=0$, is selected (in Fig. 3 it would be a Pass primitive slightly faster than $m_{2}$ ).
} 
shows three. Hence, we need a mechanism for conflict resolution because actions that are possible for one object in isolation may not be compatible with another.

To solve this problem, evolution has provided vertebrates with action-selection mechanisms implemented in old evolutionary brain structures: the basal ganglia [44]-[46].

We can take inspiration from this natural architecture and set up a similar mechanism for the artificial co-driver. For this we borrow three ingredients from natural action-selection mechanisms: 1) representation of actions in metric spaces, so that close actions are close points in that space, 2) an inhibition mechanism so that actions that are not feasible for one object can veto similar (close) actions that might be feasible for another object and 3) a weighting mechanism so that we can select among the non-inhibited actions.

1) Action Representation: From (A1.3), (A1.8), we note that the first two coefficients of the motor primitive polynomials, $c_{1}=v_{0}$ and $c_{2}=a_{0}$, encode the initial state, and are thus the same for all primitives.

Conversely, the last three coefficients $c_{3}, c_{4}, c_{5}$, together with movement time $T$, encode the goal state, and thus vary among primitives. Goals and final states can hence be represented in the four-dimensional space formed by $c_{3}, c_{4}, c_{5}, T$.

We argue that, for the purpose of comparing actions, the action space $c_{3}, c_{4}, c_{5}, T$ can be conveniently projected on the 1-D $c_{3}$ subspace. This projection is represented at the bottom in Fig.3 and Fig.4. Note that $c_{3}$ is the initial jerk $\left(j(0)=c_{3}\right)$ of the primitive.

The justifications for this reduction of the dimensionality of the action space move from the consideration that $j(t)$ is the control input of the plant model (A1.1). That is, the driver acts by controlling the rate of change of the acceleration:

$$
j(t)=c_{3}+c_{4} t+\frac{1}{2} c_{5} t^{2}, \quad t \in[0, T]
$$

In a receding-horizon conceptual framework -such as when motor plans are continuously updated to adapt to the changing environment $-j(0)$ is the instantaneous control. If we imagine two different goals, enacted by two distinct motor primitives sharing the same $c_{3}$, they require the same instantaneous control (the control input will become distinct only later, as the horizon rolls). Hence, projection on the 1-D $c_{3}$ subspace means that actions that share the same initial control are lumped together.

2) Inhibition Mechanism: Let us assume that one of the actions lumped at a given $j(0)$ is hazardous. When operating with short horizons (like in this application) we may suppose that this very condition inhibits all other actions that share the same $j(0)$; i.e., a hazardous action blocks all actions that would be produced with the same instantaneous control.

Fig.5 gives one example for the situation depicted in Fig.1, right. From top to bottom, it shows the individual action spaces for the traffic light, for the first vehicle and for the second vehicle.

If we consider the three objects (vehicles and traffic light) in isolation, we see that for the traffic light it is possible to either stop or pass within the shown intervals; for the first
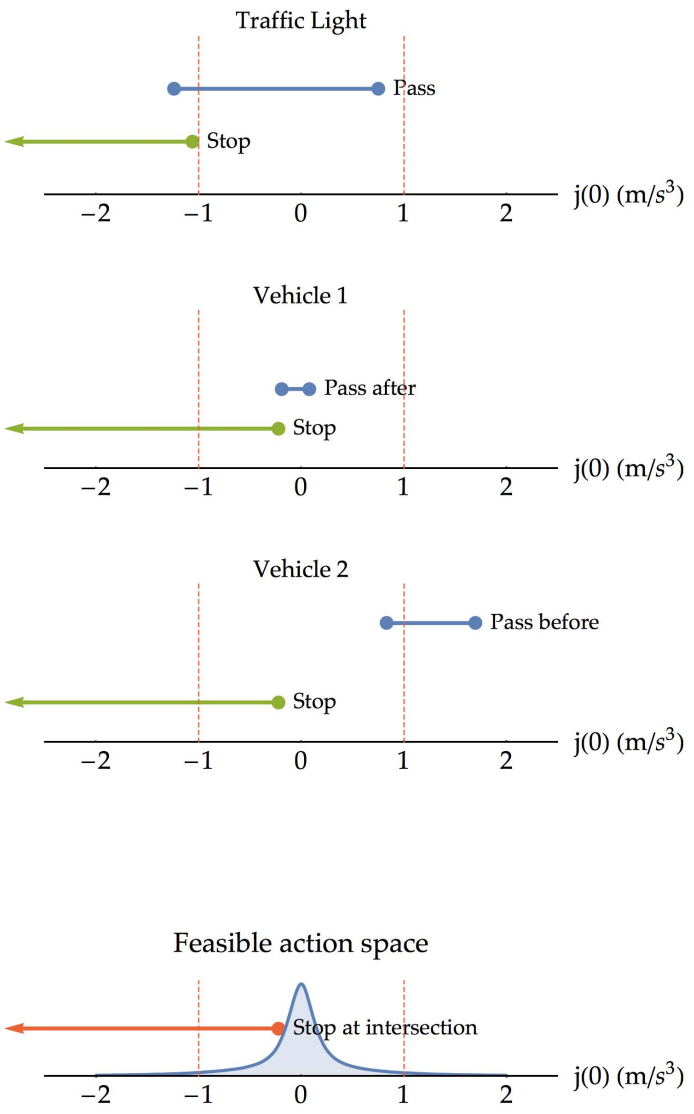

Fig. 5. Top 3 charts: representation of actions compatible with traffic light, vehicle 1 and vehicle 2 of Fig.1 right, when considered in isolation. Bottom chart: actions that are compatible with the three together are represented by the intersection of the three action spaces (in the example the orange jerk interval extending to $-\infty$ ). The bell shaped filled curve represents the experimental distribution of longitudinal jerk for an average human driver in urban scenarios nearby intersections and roundabouts. The most likely action is the right extreme of the interval, shown with the orange dot. Tracing this action back in the three above charts, one can realize that it corresponds to passing the traffic light and stopping at the intersection with the vehicles. The two dashed vertical lines at $-1 \mathrm{~m} / \mathrm{s}^{3}$ and $+1 \mathrm{~m} / \mathrm{s}^{3}$, represent the interval enclosing about $90 \%$ of human directed jerk. If there were no feasible action within this conventional interval an advisory warning would be produced (only $5 \%$ probability that the human driver is going to act). Similarly, the interval $-3 \mathrm{~m} / \mathrm{s}^{3}$ and $+3 \mathrm{~m} / \mathrm{s}^{3}$ (not shown), represents $99 \%$ of human directed jerk. If no feasible action is found within the latter a cautionary warning is produced.

vehicle, it is possible to stop at the intersection or pass after the vehicle; for the second vehicle, it is possible to stop or pass before.

However, when we consider the three objects together, feasible actions are only those shown in the bottom chart, i.e. the intersection of the actions that are possible for each object separately, which is represented by the orange interval extending from the dot at about $j(0) \approx-0.2 \mathrm{~m} / \mathrm{s}^{3}$ towards $-\infty$.

Of course, the procedure that has been described here for the example of Fig.1 right, with three threats, works for any number of intersecting vehicles (with any number of intersections) and any number of traffic lights. It is sufficient to compute the action spaces of all the objects before making the intersection space. 
3) Mirroring: In the bottom chart of Fig.5, the bell shaped filled curve represents the experimental distribution of longitudinal jerk for an average human driver, which was obtained in the InteractIVe project, for stop and yield scenarios (see Appendix 2).

The most likely action is the right extreme of the interval, shown with the orange dot. Tracing this action back in the three above charts, one can realize that it corresponds to passing the traffic light and stopping at the intersection with the vehicles. The two dashed vertical lines at $-1 \mathrm{~m} / \mathrm{s}^{3}$ and $+1 \mathrm{~m} / \mathrm{s}^{3}$, represent the interval enclosing about $90 \%$ of human directed jerk (Appendix 2). If there were no feasible action within this conventional interval an advisory warning would be produced (only $5 \%$ probability that the human driver was going to act in either way). Similarly, the interval $-3 \mathrm{~m} / \mathrm{s}^{3}$ and $+3 \mathrm{~m} / \mathrm{s}^{3}$, represents $99 \%$ of human directed jerk. If no feasible action is found within the latter interval a cautionary warning is produced (we use the first criterion when mirroring a correct driver and the second criterion when mirroring a slightly incorrect driver - see section IV.B).

\section{SySTEM EVALUATION}

\section{A. Theoretical Warning Time}

Perhaps, the most important feature of a preventive safety system, is providing warnings with sufficient anticipation, to allow room for the driver reaction time plus the time needed for the corrective maneuver.

If we consider the case where only stop is possible, e.g., as in Fig.5, the warning time can be derived analytically.

Following section V.B.3, warning is produced when there is no action within the interval $\left[-j_{t h}, j_{t h}\right]$, i.e., when:

$$
j(0)<-j_{t h}
$$

Combining (A1.4) with (A1.3), the above condition yields the distance $d_{w}$ at which the warning is generated (condition (A1.5) is always satisfied in this case):

$$
d_{w}=\frac{9 a_{0}^{3}+18 a_{0} j_{t h} v_{0}+\sqrt{3} \sqrt{\left(3 a_{0}^{2}+4 j_{t h} v_{0}\right)^{3}}}{10 j_{t h}^{2}}
$$

Inspection of (6) reveals that the warning distance, besides being a function of vehicle speed $v_{0}$ and threshold $j_{t h}$, is also a function of the current acceleration $a_{0}$. To envision this, Fig.6 plots the time to intersection $\left(T T I_{w}=d_{w} / v_{0}\right)$ of the theoretical advisory warning $\left(j_{t h}=1 \mathrm{~m} / \mathrm{s}^{3}\right)$ as a function of velocity $v_{0}$ for different values of vehicle acceleration $a_{0}$.

For example, at the uniform speed of $50 \mathrm{~km} / \mathrm{h}$ the warning (6) is produced at $T T I_{w}=5.2 \mathrm{~s}\left(d_{w}=72 \mathrm{~m}\right)-$ which leaves some room for corrective actions after the idle reaction time. In case the driver does not react, a cautionary warning ( $j_{t h}=3 \mathrm{~m} / \mathrm{s}^{3}$ ) would be produced according to (6) at $T T I_{w}=3 s\left(d_{w}=41.4 m\right)$.

If the vehicle were accelerating at $a_{0}=1 \mathrm{~m} / \mathrm{s}^{2}$, the advisory warnings would be anticipated at $T T I_{w}=7.5 \mathrm{~s}\left(d_{w}=\right.$ $104 \mathrm{~m}$ ) and the cautionary warning would happen at $T T I_{w}=$ $3.7 \mathrm{~s},\left(d_{w}=51 \mathrm{~m}\right)$.

For large negative values of the acceleration $\left(a_{0}<-2 \div\right.$ $\left.-3 \mathrm{~m} / \mathrm{s}^{2}\right)$, and at low speed $\left(v_{0}<30 \div 50 \mathrm{~km} / \mathrm{h}\right)$, the warning

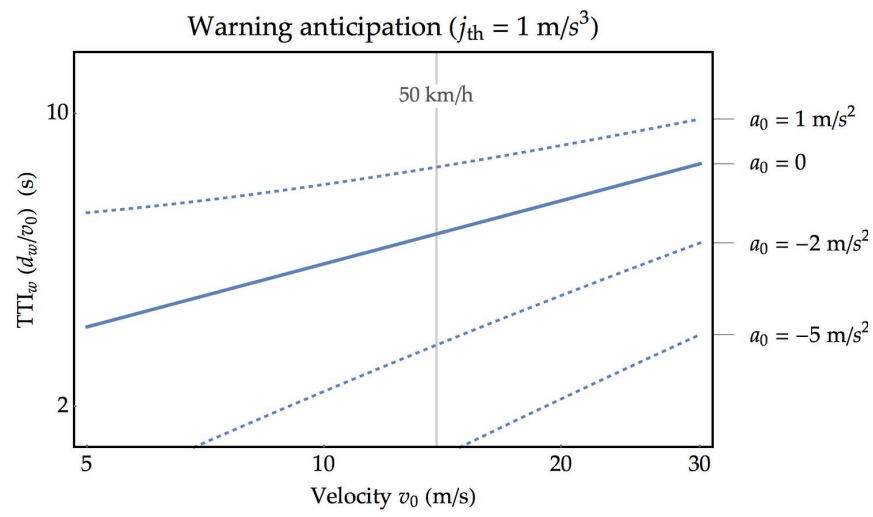

Fig. 6. Theoretical anticipation of the advisory warning $\left(j t_{h}=1 \mathrm{~m} / \mathrm{s}^{3}\right)$ as function of velocity and acceleration. The chart shows the warning distance divided by vehicle speed $\left(T T I_{w}\right)$ as function of vehicle speed and for different values of vehicle acceleration. Note that the time to actually stop, if the vehicle brakes, is longer than $\left(T T I_{w}\right)$.

anticipation $T T I_{w}$ may be less that $2 \mathrm{~s}$. However, in this case the vehicle is already braking, which means that the driver only needs to increase the braking effort. Moreover, because the vehicle is decelerating, the time it will actually take to reach the stop point is greater than $T T I_{w}$. For example, at $a_{0}=-3 \mathrm{~m} / \mathrm{s}^{2}$ and $v_{0}=45 \mathrm{~km} / \mathrm{h}$ the advisory warning is produced at $T T I_{w}=2.02 \mathrm{~s},\left(d_{w}=25.2 \mathrm{~m}\right)$. However, at the current acceleration the vehicle would actually stop in $26 . \mathrm{m}$ and $4.16 \mathrm{~s}$; hence trespassing the stop line by less than $1 \mathrm{~m}$ and in nearly twice the $T T I_{w}$ time (hence with still some time left for correction).

\section{B. Testing Against a Naturalistic Driving Dataset}

In the EU FP7 InteractIVe project a user test campaign was carried out, involving 25 test persons driving twice on a $\sim 40$-minute loop in urban, extra-urban and motorway roads near Turin in Italy. The drivers all made the same course in public roads with other traffic. However, the other vehicles were not communicating and there were no smart structures. Hence this dataset can only be used to extract samples of real driving in intersection stop locations (localization was available with digital maps) for testing the stop warning algorithms, such as no warning should be produced in maneuvers that happen to stop and sufficient anticipation should be produced in maneuvers that did not stop. A second experimental set is thus presented below to test the system as a whole.

The InteractIVe vehicle collected a particularly rich set of data from on-board vehicle state sensors, GPS and digital maps localization, range sensors detecting objects, camera for object classification and lane detection. Data fusion algorithms have been used to improve data and state estimation, finally producing detailed information such as, e.g., accurate vehicle speed, acceleration, longitudinal jerk estimation, position relative to the road, lane geometry, position and speed of the other vehicles, brake, gas pedal and steering wheel commands etc.. This dataset has been already described in previous papers which the reader can consider for more details [11], [47]-[49].

To test the algorithms of this paper, portions of driving data have been isolated in the vicinity of 16 stop and 

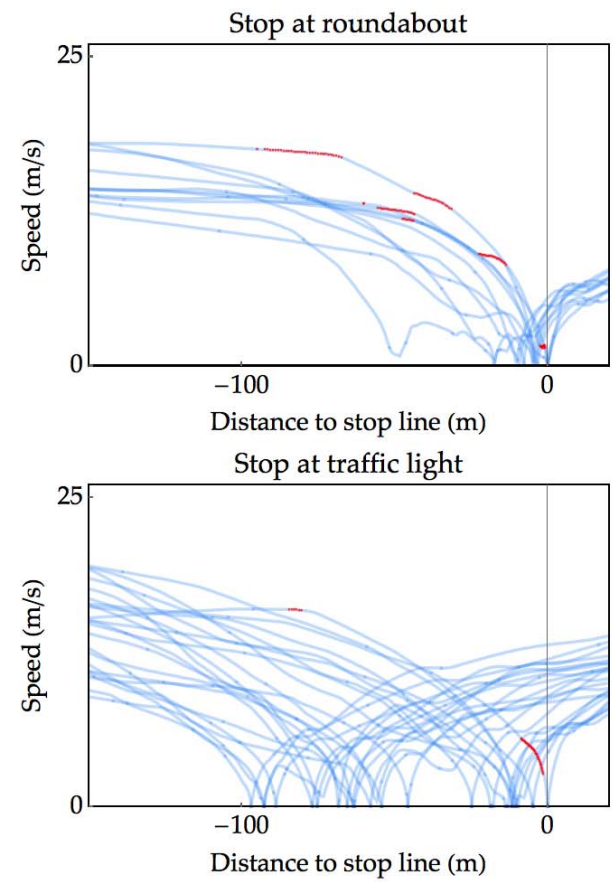

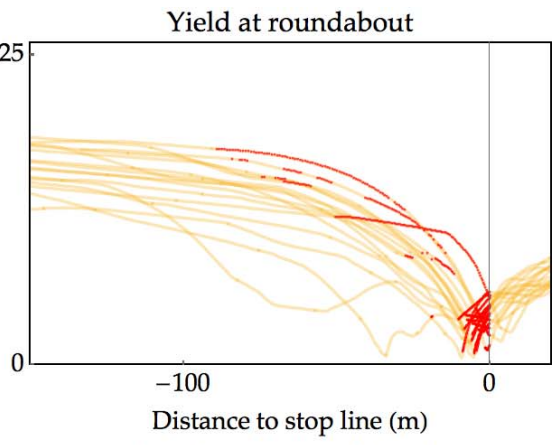

Possible stop at traffic light

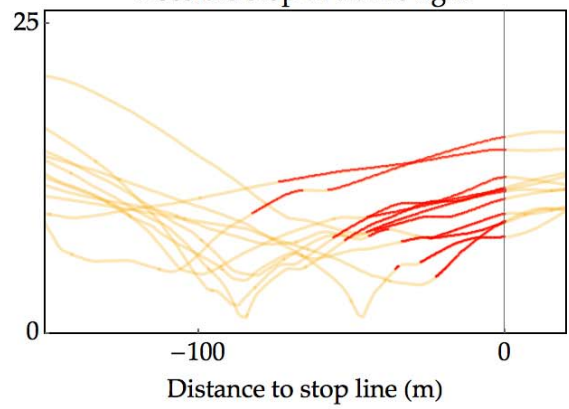

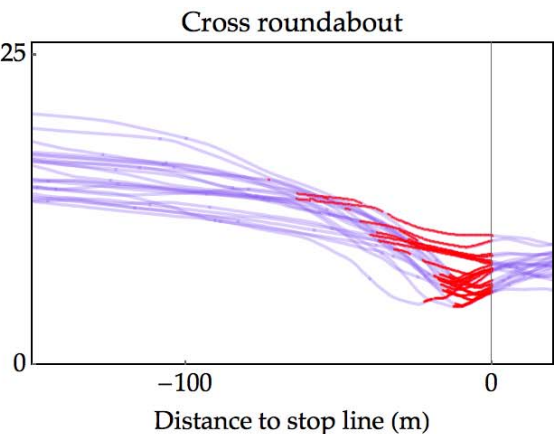

Distance to stop line $(\mathrm{m})$

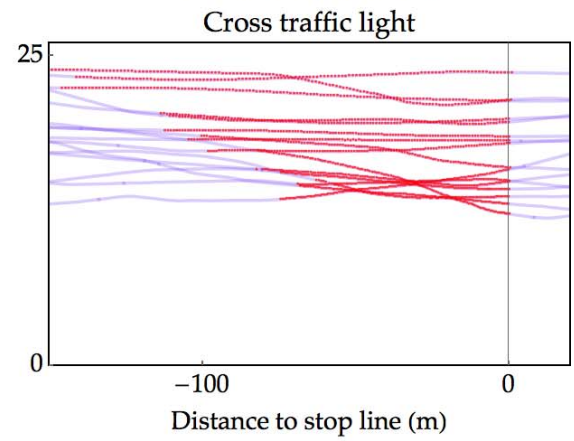

Fig. 7. Typical driver behaviors observed in the InteractIVe project, in roundabouts (top) and intersections with traffic light (bottom). Maneuvers ending with stop are shown at the left. Maneuvers with speed reduction for yield (top) or queues (bottom) are shown at the center. Maneuvers with unaffected speed (bottom) or speed adapted to the curvature (top) are shown at the right. The red dots show where any advisory warning would be produced in the hypothesis that the vehicle should stop. The fastest approaches to the stop line (left) may generate advisory warning which disappear when the driver corrects the acceleration. Warnings would also be produced when the driver resume speed (center) or get close enough to the stop line (right). More in the main text.

yield locations; spanning a few hundred meters, from the beginning of the approach maneuver before the stop point, to the recovery of the steady state speed after it [49]. A total of about 800 maneuvers have been obtained in this way.

Fig.7 shows two example locations that well represent the whole set: a roundabout (top) and a traffic light (bottom). Plots representing the speed behaviors of different drivers are shown, splitting situations when drivers stopped (left), or reduced speed for yield or possible stop (center) or crossed without particular speed reductions (right). ${ }^{4}$ The left case (stop) reveals that vehicles often stop in queues at some distance from the stop line (especially for the bottom scenario). The center case (yield) shows that yield trajectories initially are very similar to the stop ones -because drivers are preparing for a possible stop- from which they depart only later, when they can assess that passing is definitely possible, and resume speed. The right case (cross) is the simplest one, where the driver's intention is maintained for the entire maneuver.

The red dots in Fig.7 mark points where the stop primitive would require $j(0)$ outside the interval $\left[-j_{t h}, j_{t h}\right]$ for the advisory warning. This means that, unless crossing were possible, an advisory warning would be produced.

Let us first consider stop at the traffic light. The bottom-left case shows two borderline situations that produce warnings. The first happens at about $90 \mathrm{~m}$ from the stop line when the driver is travelling at nearly uniform speed and faster than all others at that point. The second happens close to the stop

\footnotetext{
${ }^{4}$ In roundabouts speed is always reduced because of the curvature of the road.
}

line, when the driver is again the fastest and closest to the stop line, whilst he/she is braking, but not enough. In both cases the drivers made a correction just immediately after the time the system would have issued the warning. The alarm would disappear after driver corrections. The drivers would clearly perceive these alarms as related to the combination of high speed and insufficient deceleration in relation to the distance. The fact the warnings disappear as soon as the acceleration is corrected reinforces this interpretation.

The two drivers might actually be more aggressive than the average driver implicit in the system design, and they might disagree with the system, considering the warnings to be excessively cautious. Indeed, the threshold $j_{t h}=1 \mathrm{~m} / \mathrm{s}^{3}$ has been set in relation to the distribution of human directed jerk for the whole driver cohort (Appendix 2). Following Appendix 2, one might however collect specific driver's distributions and set personalized thresholds. It is worth noting that allowing some trespassing tolerance (about 4 meters in this case) both warnings would disappear even without personalization. Hence the fact that very often vehicles stop before the line significantly contributes to the reduction of false alarms.

The roundabout is a scenario with potentially more false alarms (top, left). The reason is that drivers approaching a roundabout have some expectation to find it 'crossable' (i.e., there is some prior expectation for the 'pass' affordance). As a consequence, some drivers might drive for crossing, and rely on corrections when crossing turns out to be impossible. This altered state is detected by the co-driver with the issue of the alarms. 

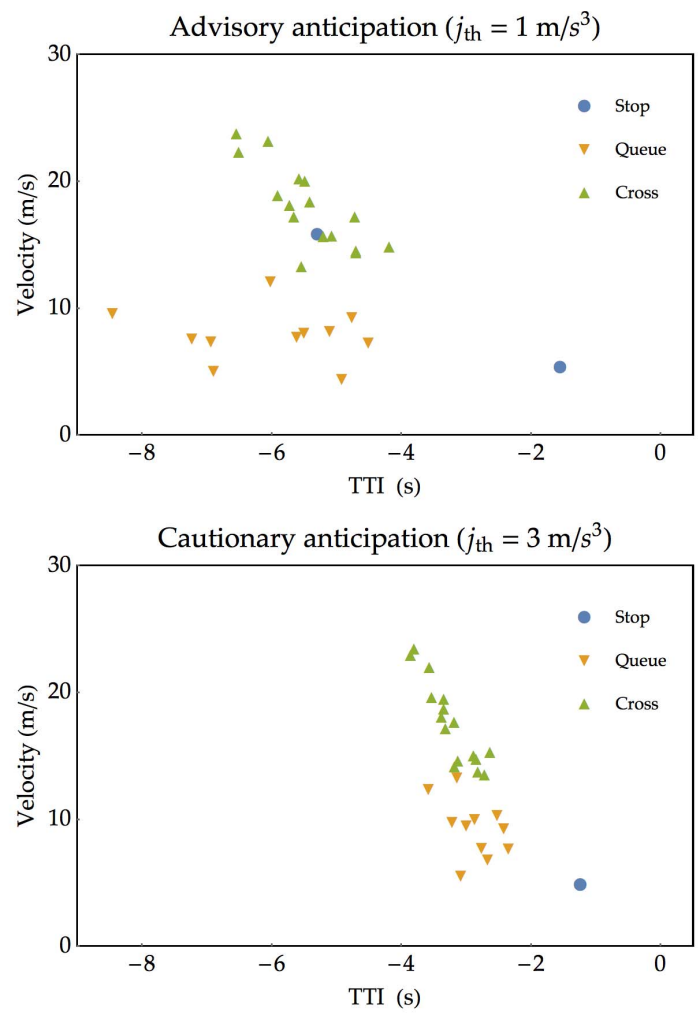

Fig. 8. Anticipation (TTI) of Advisory and Cautionary warnings in the traffic light scenario. Advisory warnings are given with more the $4 \mathrm{~s}$ anticipation (increasing with speed and with acceleration). Cautionary warnings are given with 2.5-4.5 $\mathrm{s}$ anticipation (again increasing with speed and acceleration).

If we now consider the center and right sub-cases, we see that, sooner or later, crossing is always detected. Fig.8 plots speed versus TTI for advisory and cautionary warnings of the traffic light scenario. Except for one point (the late braking mentioned above), advisory warnings are issued with more than 4 seconds of anticipation; increasing with speed for the cross scenario and increasing with acceleration (not seen in charts) for the yield/queue scenario. Similarly, cautionary warnings have more than 2 seconds of anticipation.

\section{Discussion and Comparison With the Literature}

As shown in the following, comparison with the literature is not as straightforward as it might seem at a first glance. Perhaps the closest system is the one described by Aoude [22], and developed within the CICAS project. However, there are many caveats.

Methodologically, the system in [22] presents two Machine Learning (ML) applications (HMM and SVM-BF) that classify incoming vehicles into compliant and violator classes. Conversely the agent of this paper is an artificial cognitive system implementing mirroring mechanisms, which infers human intentions among many humanlike generated affordances. Following Demiris [8] and Csibra [10], the latter is thus an example of generative approaches, whereas the former two work the other way round.

Comparing a generative approach to a classification approach is not straightforward for at least two reasons: a) an agent that implements mirroring is dynamic, and interacts with the driver. As noted, Fig.7 shows that a warning is issued when a driver is approaching the stop line fast, but is cancelled when the driver corrects the trajectory. The notion of 'false alarm' hence somewhat fades in the interaction between human and artificial agents (the human may think that the agent is too conservative, but anyway capable of 'understanding' his actions); $b$ ) human behavior is adaptive: intentions may change at any time (e.g., Fig.7 showed that in a yield situation drivers switch from stopping to crossing) and this variability of intentions does not fit well into the conceptual framework of static classification (at least rolling classification would be necessary, such as in [23]).

For what concerns the application domain, the two ML applications [22] operate only in signalized intersection scenarios,${ }^{5}$ in which they have been trained and their output is binary (compliant vs. non-compliant). Training was not trivial, using very large number of training samples. Scaling to other scenarios, such as multi-classification in yield scenario is also non-trivial, in practice requiring to re-engineer the system [23]. Conversely, the agent of this paper, because of the implicit driver model, is more general. Its output may be as vast and articulated as the library of motor behaviors the agent is aware of, as shown for example in the discussion of Fig.5. Moreover, extension of the library of actions almost automatically makes the agent capable of understanding new goals. ${ }^{6}$ Observation of other road users -the importance of which has been recognized in [23]- is also implicit, and, unlike [23], any number of such external agents can be accommodated by the inhibition mechanism in section V.B.2. Parametrization of the agent is carried out using naturalistic driving data, and is facilitated because it is constrained by many principles that hold for human sensorimotor control. The modelling approach that is implicit of the agent, finally provides insights that are not given by the ML approaches, as noted with Fig.7.

The two ML methods [22] carry out classification either at TTI $=2 \mathrm{~s}, 1.6 \mathrm{~s}$ or $1 \mathrm{~s}$. For example, at TTI $=2 \mathrm{~s}$ the SVM method achieves $65 \%$ true positive rate with $5 \%$ false positive rate. A precise comparison with these figures is not immediately feasible for several reasons: $a$ ) the original trajectories data used in [22] are not public for testing; b) as shown in Fig.7, there are at least three categories of approaching trajectories (aimed at stop, yield or cross) and, in the first one, the actual stop may occur before the stop line. Hence, it is difficult to use our data to set up comparable situations to derive true and false alarm rates. Nonetheless, grossly speaking the stop situation described in Fig.7, bottom, shows two 'false' alarms. As shown in Fig.8 any of the other maneuvers, assuming they were incorrect (i.e., the driver missing to recognize he/she has to stop), would produce advisory warnings with 4.5-8.5 $\mathrm{s}$ anticipation and cautionary warnings with 2.5-4.5 s anticipation. Hence no miss alarm (no alarm with less than $2 \mathrm{~s}$ anticipation in this comparison) would occur;

\footnotetext{
${ }^{5}$ It is worth noting that while this is the most frequent scenario in the US in the EU the vast majority of trajectory intersections occur in roundabouts.

${ }^{6}$ This corresponds to learning new behaviors in live beings.
} 
and sensitivity (true positive rate) would be $100 \%$. Estimation of false positive rate for the advisory warning is 3 out of 44 ( 2 false alarms out of 23 stop maneuvers in Fig.7, bottom, and 1 in 21 maneuvers in another traffic light that is available in the Interactive dataset). For cautionary warnings, the count drops to 1 out of 44 . However, as previously discussed, these alarms occur in borderline situations, just before the driver acts on acceleration, and are dynamically cancelled as soon as acceleration is reduced. Hence, we argue that the conceptual framework of binary categorization into 'false' and 'correct' alarm is too sharp for driver-robot dynamic interactions.

Another aspect that has to be commented is the warning time: in addition to the reaction time, it should allow enough time for drivers to implement some corrective maneuver. ${ }^{7}$ According to [50], the brake reaction time to requests from driver assistance systems depends on many factors (level of surprise, experience with the system, trust in the system, whether two alert levels are implemented, design of the human machine interface, etc.). For example, for an unexpected stimulus the brake reaction time may be $\sim 1.28 \mathrm{~s}$ mean with $0.2 \mathrm{~s}$ standard deviation, which means $1.54 \mathrm{~s}$ to capture $90 \%$ of users. If the system does not include an advisory level, brake reaction time to the 'first stimulus' may degrade to $\sim 1.39 \mathrm{~s}$ mean with $0.47 \mathrm{~s}$ standard deviation, which means $\sim 2 \mathrm{~s}$ to capture $90 \%$ of users. Things are likely to further worsen in an intersection scenario, where obstacles may be out of sight, requiring the driver to seek some preliminary visual confirmation (increasing the gaze response time component of the reaction time). These considerations indicate that a two-level alert system with anticipations of $\sim 1.6 \mathrm{~s}$ barely allows room for the reaction time of the 90-quantile driver, and additional time for action should definitely be sought. Indeed, the informal opinions reported by our test drivers (see next section) were that $\sim 4 \mathrm{~s}$ anticipation was considered to be 'barely sufficient' to act. These were our main motivations to seek the anticipations shown in Fig.8

A final consideration that can be made is that there may be cases that can be decided very early (for example if the driver misses to recognize a stop sign and proceeds as in Fig.7, right). A system that dynamically mirrors the driver, can issue alarms as soon as the dangerous situation occurs, without needing to wait for the last classification time (a rolling classifier may work).

\section{Dedicated Experimental Campaign}

The agent of this paper has been finally tested using two real vehicles and one traffic light, all customized with communication channels.

The two vehicles used no sensor other than the Global Positioning System of the prospective e-call system (with Real Time Kinematic correction), the on-board standard equipment accelerometers, gyro-meter and odometer, and digital maps.

Each vehicle transmitted its own position, velocity and acceleration, and a description of a stretch of the road ahead represented with a list of up to 20 arcs- with a typical horizon

\footnotetext{
${ }^{7}$ The system in [22] is intended to warn other drivers for a potential violator, but these may be as close as the violator to the intersection.
}

of up to $200 \mathrm{~m}$. Transmission rate was $100 \mathrm{~ms}$. Computation time was neatly shorter and fitted well within the $100 \mathrm{~ms}$ cycle time.

The custom traffic light transmitted its position, current phase and the times and phases of the next 3 .

After receiving information from the other vehicle and the traffic light each vehicle located the traffic light and the road of the other vehicle on its own road, finding the intersection of the lanes $c$ and estimating the position of the stop and clear points $s$ and $l$ (Fig.10, left).

Professional drivers tested the system as follows:

1) Traffic Light Use Case: One vehicle was driven on a private road with programmed traffic light cycles to test the following situations:

a) Crossing the traffic light in permanent green phase, with and without complying with the speed limit (in the latter case obtaining alarms).

b) Starting when the traffic light turns green (no alarms had to be produced).

c) Crossing the traffic light near the switching from green to yellow phase. While no alarm was produced when crossing before, a predictive alarm was expected, and obtained, when crossing was going to happen during the grace time of the yellow phase (this case is commented below).

d) Crossing the traffic light in the red phase (or at the yellow phase with grace time expired). In this case, the sequence of advisory and cautionary warnings was observed with times in agreement with (6).

e) Crossing the traffic light near the switch from red to green phase. The sequence of advisory and cautionary warnings was observed when the vehicle was going to cross in the red phase. However, no alarm was generated when the vehicle was going to cross with the green light, even if the traffic light was still displaying red (this case is commented below).

$f$ ) Driving in compliance with the traffic light and stopping 'naturally' at the red light (no warning was observed; here considerations made for Fig.7 apply).

$g$ ) Initially ignoring the red traffic light, to brake only after receiving the cautionary alarm. This was intended to simulate, to some extent, a driver mistake and the following emergency brake maneuver (this case is commented below).

While a detailed analysis of all the above cases is beyond the possibility of this paper, we comment here the most salient situations, also because some unexpected aspects emerge, which have an impact on human factors.

Fig.9, top, shows one situation that happened for case $c$, i.e., when crossing the traffic light at the onset of the yellow phase. The figure plots the longitudinal acceleration (the initial velocity is $48.8 \mathrm{~km} / \mathrm{h})$ and the warning level $(1=$ no alarm, $2=$ advisory, 3 = cautionary) as function of the current Time-To-Intersection (TTI). The colored bar on top represents the current traffic light phase (green till $-1 \mathrm{~s}$, then yellow). The initial speed, say at e.g., TTI $\sim 6 s$, would have been sufficient to cross the traffic light with the green (perhaps using a slight acceleration). However, the vehicle decelerates and at 

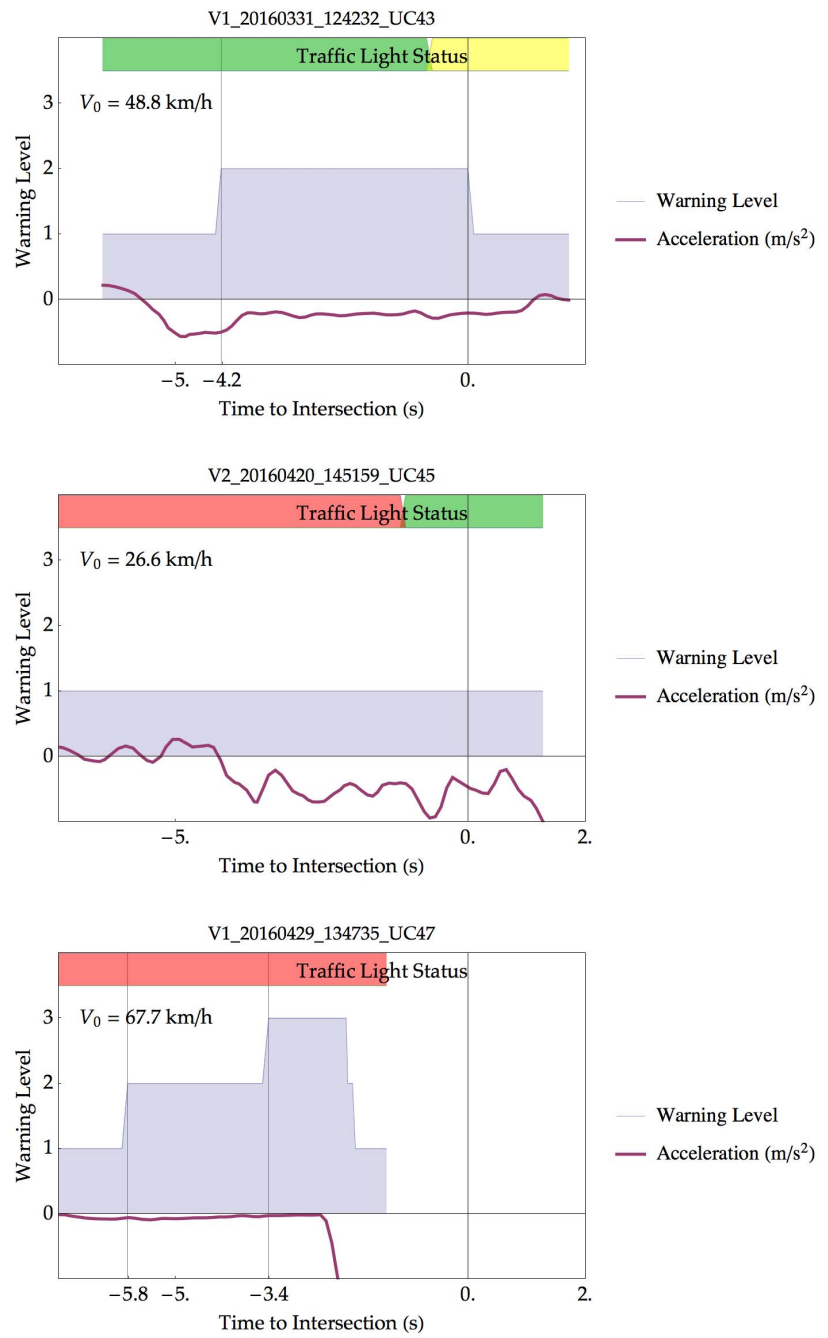

Warning Level

- Acceleration $\left(\mathrm{m} / \mathrm{s}^{2}\right)$

Fig. 9. Three examples of testing with a Traffic Light with phase communication. Top: the vehicle approaches a traffic light in the green phase. The phase will switch to yellow about $1 \mathrm{~s}$ before crossing. At TTI $=4.2 \mathrm{~s}$, the system preventively detects crossing with the expired green and issues a level 1 alarm. Center: the traffic light is red but the driver is going to cross in the next green phase. No warning is produced (see discussion). Bottom: the driver was asked to brake only after receiving the cautionary warning.

$T T I=4.2 \mathrm{~s}$ an advisory warning is issued, because, due to the reduced speed, the vehicle will reach the intersection with green expired. Indeed, the crossing occurs at about 1 second in the yellow phase. After crossing the alarm disappears.

Fig.9, center, shows a more interesting case. Here the driver approaches the traffic light which is red; but the system knows it will turn green $1.5 \mathrm{~s}$ before crossing, hence a 'pass' maneuver, as in Fig.5, exists and no alarm is issued. From the human factor point of view, however, the driver is approaching a red traffic light without warning from the system. Hence, a better design might be to ignore the future green status until it becomes visible.

Fig.9, bottom, concerns case $g$. The driver approaches a red traffic light and was instructed to ignore the advisory warning and react only after perceiving the cautionary warning. At $67.7 \mathrm{~km} / \mathrm{h}$ the advisory warning is produced at TTI $=5.8 \mathrm{~s}$ and the cautionary warning at $3.4 \mathrm{~s}$. The driver (a professional driver) reacts in about $0.9 \mathrm{~s}$ and stops the vehicle before
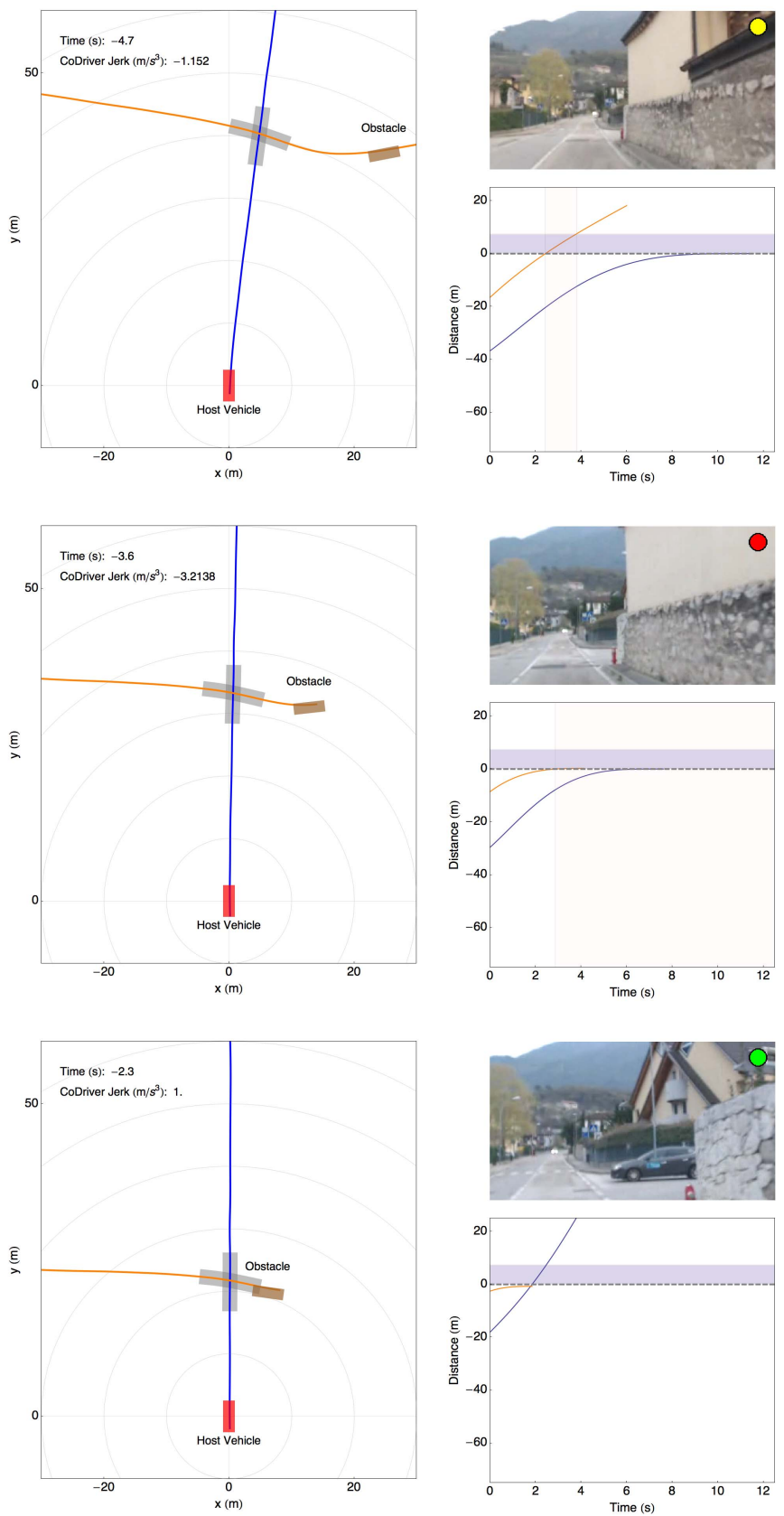

Fig. 10. One example of warnings produced for an obstacle out of sight. Top: advisory warning triggered at TTI $=4.7 \mathrm{~s}$. Center: cautionary warning triggered at TTI $=3.6 \mathrm{~s}$. Bottom: the obstacle is visible when the dangerous situation is elapsed. More details in the main text.

the line. However, there was no surprise and the traffic light was in the field of view of the driver.

2) The Intersection Use Case: Two vehicles with vehicle-tovehicle communication were driven in a selected intersection for which a digital map was available. Two approaching directions were blind (a high wall occluded the view) and two allowed each other vehicle sight. The drivers were instructed to create various hazardous situations as follows:

a) the two vehicles proceed on intersecting collision paths; the car that must give way stops only at the very end (this case is commented below, and a video recording is also available in the supplement material). 
b) as above except that the second car turns on the same road of the first car just in front of it. The purpose was to study the transition from intersection to car following scenario.

c) as above except that the second car turns right while the first car is arriving in the opposite direction from right. The purpose is to study the transition from an intersection to non-intersecting trajectories.

Fig.10 presents the case $a$, showing three salient moments: at $\mathrm{TTI}=4.7 \mathrm{~s}$ when the advisory warning is issued (top), at TTI $=3.6 \mathrm{~s}$, when the cautionary warning is issued (center) and at TTI $=2.3 \mathrm{~s}$ when the field of view is finally not occluded. The left sub-charts show the bird's eye view, with the host vehicle, the obstacle and the centerline of the host and obstacle lanes. The intersection region is highlighted in gray. The longitudinal co-driver jerk $j(0)$ and the time to intersection are recorded on the top left. The top right panel shows the camera view, with a colored circle inset encoding the warning level (yellow, red, green from top to bottom). Note that the intersecting vehicle is visible only in the last frame, and is hidden by a wall when the warning is issued (this is an example where a driver receives a warning which cannot be visually confirmed). The bottom right sub-charts show the predicted trajectory of the obstacle (orange line) and the co-driver planned trajectory (blue line below). The (professional) driver of the opponent vehicle was instructed to create a dangerous situation, braking only at the last time. At TTI $=4.7 \mathrm{~s}$ (Fig.10, top) the opponent vehicle is estimated to enter the intersection in about $2.5 \mathrm{~s}$ and leave it slightly before $4 \mathrm{~s}$. To respect the gap $T_{g}$ of (3), the host vehicle cannot enter before $8 \mathrm{~s}$. Hence, the co-driver choice is to stop, which needs $j(0)=-1.152 \mathrm{~m} / \mathrm{s}^{3}$ and causes the advisory warning. At TTI $=3.6 \mathrm{~s}$ (center) the opponent vehicle (in the execution of a brake maneuver) looks going to enter the intersection in about $3 \mathrm{~s}$. The co-driver maneuver is now more urgent and a cautionary warning is triggered. At TTI $=3.4 \mathrm{~s}$ (not shown) the opponent vehicle brakes enough that the co-driver predicts it will no longer enter the intersection. At this time, the warning disappears. The almost stopped vehicle is seen in the last frame (Fig.10, bottom).

\section{CONCLUSIONS}

This paper demonstrated a principled approach for the design of driver assistance systems based on 'mirroring' the human sensorimotor system.

An intersection collision warning application derived with these principles is fully documented and easily applicable. In addition, because the co-driver agent is based on a model of human motor control, its parameters can be easily interpreted, tuned with naturalistic driving data and personalized.

However, beyond the application itself, we believe the method is of more general value and the application is scalable by adding new behaviors to the layered architecture.

For example, as is, the system does not consider carfollowing situations (this paper is about intersection driver assistance). So, if the host vehicle were following a leading one which suddenly brakes, no rear-end collision warning would be generated, even in an intersection situation.

However, a new function such as rear-end collision warning can be integrated by developing motor primitives for car following with the logic of section $\mathrm{V}$ (in particular this requires defining proper final conditions for the car following goal). With these, the action space for car following (which will look like $j(0) \in\left[-\infty, j^{*}\right]$, with $j^{*}$ the longitudinal control that separates car following from collision) can be integrated in the action selection mechanism of Section V.B. Just like in biological layered control architecture, any new behavior becomes part of the agent motor repertoire because it is integrated by a unified action selection mechanism.

\section{APPENDIX 1 - MOTOR PRIMITIVES}

\section{A. Stop Motor Primitive}

The stop behavior of a human car driver has been studied in detail in [49] with experimental data. It is quite complex and made of several phases (i.e., gas release, choked gas, brake and brake release) that, to be accurately modeled, would need switching between different types of motor primitives. However, for the purpose of this paper, we can adopt a simplified approach, modelling only the main brake phase with one motor primitive. Indeed, a single motor primitive acceptably fits the last 10-15 seconds of a stop maneuver ([49], section V.B), i.e., the time when the brake is pressed. Hence, because the purpose here is only to provide warnings for incorrect or insufficient braking, this simplification is sufficient and indeed convenient.

Following the minimum square jerk criterion stated in section V.A, the stop motor primitive is produced by solving the following optimal control problem ${ }^{8}$ :

$$
\begin{aligned}
& \text { Minimize } J=\int_{0}^{T} j(t)^{2} d t \\
& \text { s.t. } \\
& \text { plant model : } \dot{s}(t)=v(t), \dot{v}(t)=a(t), \dot{a}(t)=j(t) \\
& \text { initial conditions : } s(0)=0, v(0)=v_{0}, a(0)=a_{0} \\
& \text { final conditions : } s(T)=s_{f}, v(T)=0, a(T)=0
\end{aligned}
$$

The function $s(t), t \in[0, T]$, represents the travelled distance as a function of time, $v(t)$ the velocity, $a(t)$ the acceleration and $j(t)$ the jerk (the control input).

These functions link the present state of the vehicle $v_{0}, a_{0}$ to a stop state at distance $s_{f}$. The final time $T$, i.e., how long it takes to stop the vehicle optimally, is free and part of the solution.

The solution of the optimal control problem in (A1.1) gives the motor primitive, which is a $5^{\text {th }}$ order polynomial. Note that velocity, acceleration and jerk can be obtained by derivation of (A1.2).

$$
s(t)=c_{1} t+\frac{1}{2} c_{2} t^{2},+\frac{1}{6} c_{3} t^{3}+\frac{1}{24} c_{4} t^{4}+\frac{1}{120} c_{5} t^{5}
$$

\footnotetext{
${ }^{8}$ Compared to ([49], section IV.A) the problem defined in (A1.1) neglects the minimum time term $\left(w_{T}\right.$ in equation 3$)$, and requires the final acceleration to be exactly zero. These simplifications allow obtaining much simpler equations.
} 
Where:

$$
\begin{aligned}
& c_{1}=v_{0}, c_{2}=a_{0} \\
& c_{3}=60 \frac{s_{f}}{T^{3}}-36 \frac{v_{0}}{T^{2}}-9 \frac{a_{0}}{T} \\
& c_{4}=-360 \frac{s_{f}}{T^{4}}+192 \frac{v_{0}}{T^{3}}+36 \frac{a_{0}}{T^{2}} \\
& c_{5}=720 \frac{s_{f}}{T^{5}}-360 \frac{v_{0}}{T^{4}}-60 \frac{a_{0}}{T^{3}}
\end{aligned}
$$

The optimal stop time is:

$$
T=\frac{10 s_{f}}{2 v_{0}+\sqrt{4 v_{0}^{2}+5 a_{0} s_{f}}}
$$

For negative values of $a_{0}, s_{f}$ cannot be arbitrarily large. The condition that the radical in the denominator of (A1.4) must be real gives the maximum stop distance of one motor primitive with initial negative acceleration ${ }^{9}$ :

$$
s_{f} \leq \frac{4 v_{0}^{2}}{-5 a_{0}}
$$

From equations (A1.2)-(A1.4) a convenient way to represent a motor primitive is collecting the coefficients of the polynomial (A1.2) and the movement time in one vector like:

$$
m=\left\{c_{1}, c_{2}, c_{3}, c_{4}, c_{5}, T\right\}
$$

Hence, we can say that the computation of one motor primitive (and its representation) consists in the evaluation of the vector (A1.6) and we may concisely indicate this computation with the notation:

$$
m=\operatorname{Stop}\left(a_{0}, v_{0}, s_{f}\right)
$$

which means that, if $4 v_{0}^{2}+5 a_{0} s_{f} \geq 0$, the coefficients $c_{i}$ and the movement time $T$ are computed according to (A1.3) and (A1.4); and the vector (A1.6) is returned. However, if $4 v_{0}^{2}+5 a_{0} s_{f}<0, s_{f}$ is replaced by the maximum stopping distance allowed by (A1.5) before computation of (A1.3) and (A1.4). For maximum clarity, the algorithm is as follows.

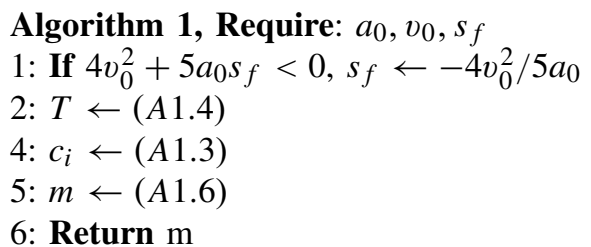

One example of a stop maneuver is the curve labeled $m_{1}$ in Fig.3. The definition in (A1.7) coincides with [49] for $w_{T}=0, w_{A}=\infty$ there.

\section{B. Primitive for Trespassing a Given Point}

Let us consider the problem of passing a given point, $s_{f}$ within a given interval of time $T \in\left[T_{1}, T_{2}\right]$ and, also, with the velocity $v_{f}$ constrained between a maximum and a minimum value $v_{f} \in\left[v_{\min }, v_{\max }\right]$.

To derive this primitive, we change the final condition for the velocity to $v(T)=v_{f}$ in (A1.1). The solution of this

\footnotetext{
${ }^{9}$ Stopping farther is still possible, but requires chaining more primitives, e.g, slowing the vehicle at some non-zero speed, resuming speed and finally stopping.
}

new optimal control problem is still represented by polynomial (A1.2), but the coefficients $c_{i}$ now read:

$$
\begin{aligned}
& c_{1}=v_{0}, c_{2}=a_{0} \\
& c_{3}=60 \frac{s_{f}}{T^{3}}-36 \frac{v_{0}}{T^{2}}-9 \frac{a_{0}}{T}-24 \frac{v_{f}}{T^{2}} \\
& c_{4}=-360 \frac{s_{f}}{T^{4}}+192 \frac{v_{0}}{T^{3}}+36 \frac{a_{0}}{T^{2}}+168 \frac{v_{f}}{T^{3}} \\
& c_{5}=720 \frac{s_{f}}{T^{5}}-360 \frac{v_{0}}{T^{4}}-60 \frac{a_{0}}{T^{3}}-360 \frac{v_{f}}{T^{4}}
\end{aligned}
$$

At this point, instead of computing the optimal movement time $T$ for the given boundary conditions -similarly to what was done for (A1.4)-, we reverse the logic and express the final velocity $v_{f}$ in terms of the movement time $T$ :

$$
v_{f}=\frac{15}{8} \frac{s_{f}}{T}-\frac{a_{0} T}{8}-\frac{7 v_{0}}{8}
$$

We obtain a parameterization of the motor primitive in $T$, which would allow immediately imposing the condition $T \in\left[T_{1}, T_{2}\right]$. In fact, for any $T \in\left[T_{1}, T_{2}\right]$ we could compute the final velocity with (A1.9) and the motor primitive with (A1.8).

However, we also need to impose the condition $v_{f} \in$ $\left[v_{\min }, v_{\max }\right]$, i.e.:

$$
v_{\min }<v_{f}<v_{\max }
$$

Substituting (A1.9) in (A.10) and given that $s_{f}, T, v_{0}>0$ (but $a_{0}$ may be either positive or negative or zero) we see that there are two cases:

1) If $a_{0} \geq 0, v_{f}$ in (A1.9) is monotonically decreasing with $T$. Hence the condition (A1.10) becomes:

$$
T_{v_{\max }}<T<T_{v_{\min }}
$$

with (note that $T_{v_{\min }}>T_{v_{\max }}$ ):

$$
\begin{aligned}
T_{v_{\text {min }}} & =\frac{30 s_{f}}{7 v_{0}+8 v_{\min }+\sqrt{60 a_{0} s_{f}+\left(7 v_{0}+8 v_{\min }\right)^{2}}} \\
T_{v_{\max }} & =\frac{30 s_{f}}{7 v_{0}+8 v_{\max }+\sqrt{60 a_{0} s_{f}+\left(7 v_{0}+8 v_{\max }\right)^{2}}}
\end{aligned}
$$

The combination of condition $T \in\left[T_{1}, T_{2}\right]$ with (A1.11) yields:

$$
T \in\left[T_{1}^{\prime}, T_{2}^{\prime}\right]=\left[T_{1}, T_{2}\right] \cap\left[T_{v_{\max }}, T_{v_{\min }}\right]
$$

Hence it is sufficient to compute $\left[T^{\prime}{ }_{1}, T^{\prime}{ }_{2}\right]$ from (A1.13) and then compute the slow motor primitive $m_{1}$ using $T=T^{\prime}{ }_{2}$ in (A1.9)-(A1.8) and the fast primitive $m_{2}$ using $T=T^{\prime}{ }_{1}$. Note that the intersection in (A1.13) may be an empty set.

2) If $a_{0}<0,(\mathrm{~A} 1.9)$ is a function that has a minimum, with value $v^{*}=\frac{1}{4} \sqrt{-15 a_{0} s_{f}}-\frac{7}{8} v_{0}$ at $T^{*}=\sqrt{-15 s_{f} / a_{0}}$. Hence:

2.1) If $v^{*}<v_{\min }<v_{\max }$ there are two intervals for $T$ that satisfy (A1.10). They represent two types of motor primitives that have already been discussed in [48], [49]. Of these, we consider the first interval $T \in\left[T_{v_{\max }}, T_{v_{\min }}\right]$, which is the same as case $a_{0} \geq 0$ above (the second interval, with larger values for $T$ represents motor primitives that may have inversion of velocity). In this sub-case, the procedure is thus the same as case 1) above. 
Probability Density of Human-directed Jerk

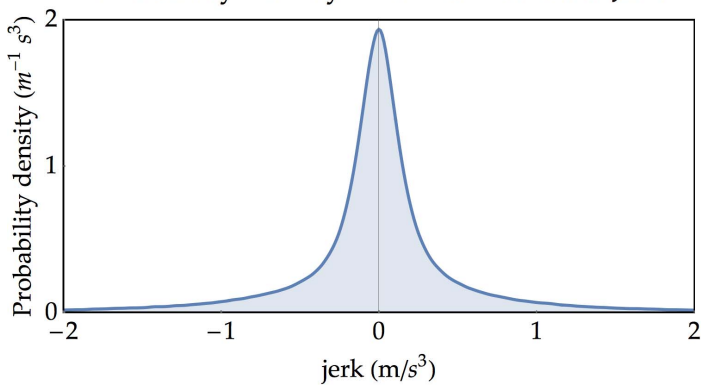

Fig. 11. Distribution of human-directed longitudinal jerk derived from 800 maneuvers (1 million observations) close to stop and yield locations. The interval $\left[-1 \mathrm{~m} / \mathrm{s}^{3}, 1 \mathrm{~m} / \mathrm{s}^{3}\right]$ accounts for $90 \%$ of observations. The interval $\left[-3 \mathrm{~m} / \mathrm{s}^{3}, 3 \mathrm{~m} / \mathrm{s}^{3}\right]$ accounts for more than $99 \%$ of observations.

2.2) If $v_{\min }<v^{*}<v_{\max }$ the solution corresponding to the first interval of 2.1 becomes $T \in\left[T_{v_{\max }}, T^{*}\right]$.

2.2) If $v_{\min }<v_{\max }<v^{*}$ there is no solution $(T \in \emptyset)$.

Here is the algorithm:

Algorithm 2, Require: $a_{0}, v_{0}, s_{f}, T_{1}, T_{2}, v_{\min }, v_{\max }$

1: If $a_{0} \geq 0$

2: $\quad\left[T_{v_{\max }}, T_{v_{\min }}\right] \leftarrow(A 1.12)$

3: Else If $a_{0}<0$,

4: $\quad$ If $v^{*}<v_{\min } \leq v_{\max }$,

5: $\quad\left[T_{v_{\max }}, T_{v_{\min }}\right] \leftarrow(A 1.12)$

6: $\quad$ Else If $v_{\min } \leq v^{*} \leq v_{\max }$,

7: $\quad T_{v_{\max }} \leftarrow(A 1.12)$

8: $\quad T_{v_{\min }} \leftarrow T^{*}=\sqrt{-15 s_{f} / a_{0}}$

9: $\quad$ Else If $v_{\min } \leq v_{\max }<v^{*}$,

10: $\quad\left[T_{v_{\max }}, T_{v_{\min }}\right] \leftarrow \varnothing$

11: $\left[T^{\prime}{ }_{1}, T^{\prime}{ }_{2}\right] \leftarrow(A 1.13)$

12: If $\left[T^{\prime}{ }_{1}, T^{\prime}{ }_{2}\right] \neq \emptyset$,

13: $\quad T \leftarrow T^{\prime}{ }_{2}, c_{i} \leftarrow(A 1.3), m_{1} \leftarrow(A 1.6)$

14: $\quad T \leftarrow T^{\prime}{ }_{1}, c_{i} \leftarrow(A 1.3), m_{2} \leftarrow(A 1.6)$

15: Else

16: $\quad m_{1}, m_{2} \leftarrow \varnothing$

17: Return $m_{1}, m_{2}$

\section{APPENDIX 2 - EXPERIMENTAL HUMAN-DIRECTED JERK IN STOP AND YIELD SCENARIOS}

Portions of driving data from the InteractIVe project have been isolated in the vicinity of 16 stop and yield locations; spanning a few hundred meters from the beginning of the approach maneuver, before the stop point, to the recovery of the steady state speed after it [49].

The longitudinal velocity, acceleration and jerk have been estimated by fusing the odometer signal and the on-board longitudinal acceleration signal with a proper Kalman filter, which was designed, among the others, to reject vehicle-drivetrain longitudinal vibrations (in the $2-3 \mathrm{~Hz}$ band) while preserving the intentional longitudinal jerk (below $\sim 0.7 \mathrm{~Hz}$ ). The latter band was found by inspection of the power spectrum of the gas pedal rate (which is the intentional action of the driver) and is in agreement with our similar findings in ([51], Fig.4) and in other literature papers, such as e.g., [52].

Fig. 11 gives the distribution of the aggregated maneuvers (1 million samples). The interval $\left[-1 \mathrm{~m} / \mathrm{s}^{3}, 1 \mathrm{~m} / \mathrm{s}^{3}\right]$ accounts for slightly more than $90 \%$ of observations. Instead, $99.3 \%$ of observations lie in the interval $\left[-3 \mathrm{~m} / \mathrm{s}^{3}, 3 \mathrm{~m} / \mathrm{s}^{3}\right]$. Distributions of the longitudinal jerk disaggregated by gas and brake pedal actions may be found in [49].

\section{REFERENCES}

[1] S. Thill, D. Caligiore, A. M. Borghi, T. Ziemke, and G. Baldassarre, "Theories and computational models of affordance and mirror systems: An integrative review," Neurosci. Biobehav. Rev., vol. 37, no. 3, pp. 491-521, 2013.

[2] G. Hesslow, "The current status of the simulation theory of cognition," Brain Res., vol. 1428, pp. 71-79, Jan. 2012.

[3] M. Jeannerod, "Neural simulation of action: A unifying mechanism for motor cognition," NeuroImage, vol. 14, no. 1, pp. S103-S109, Jul. 2001.

[4] D. M. Wolpert, K. Doya, and M. Kawato, "A unifying computational framework for motor control and social interaction," Philos. Trans. Roy. Soc. London B, Biol. Sci., vol. 358, no. 1431, pp. 593-602, Mar. 2003.

[5] J. Decety and J. Grèzes, "The power of simulation: Imagining one's own and other's behavior," Brain Res., vol. 1079, no. 1, pp. 4-14, Mar. 2006.

[6] A. N. Meltzoff, "The 'like me' framework for recognizing becoming intentional agent," Acta Psychol., vol. 124, no. 1, pp. 26-43, 2007.

[7] S. Hurley, "The shared circuits model (SCM): How control, mirroring, and simulation can enable imitation, deliberation, and mindreading," Behav. Brain Sci., vol. 31, no. 1, pp. 1-22, Feb. 2008.

[8] Y. Demiris, "Prediction of intent in robotics and multi-agent systems," Cognit. Process., vol. 8, no. 3, pp. 151-158, Sep. 2007.

[9] J. J. Gibson, "The theory of affordances," in The Ecological Approach to Visual Perception. Hillsdale, NJ, USA: Lawrence Erlbaum Associates, 1986, pp. 127-143.

[10] G. Csibra and G. Gergely, “'Obsessed with goals': Functions and mechanisms of teleological interpretation of actions in humans," Acta Psychol., vol. 124, no. 1, pp. 60-78, Jan. 2007.

[11] M. Da Lio et al., "Artificial co-drivers as a universal enabling technology for future intelligent vehicles and transportation systems," IEEE Trans. Intell. Transp. Syst., vol. 16, no. 1, pp. 244-263, Feb. 2015.

[12] J. Rios-Torres and A. A. Malikopoulos, "A survey on the coordination of connected and automated vehicles at intersections and merging at highway on-ramps," IEEE Trans. Intell. Transp. Syst., vol. 18, no. 5, pp. 1066-1077, May 2016.

[13] L. Chen and C. Englund, "Cooperative intersection management: A survey," IEEE Trans. Intell. Transp. Syst., vol. 17, no. 2, pp. 570-586, Feb. 2016

[14] L. Li, D. Wen, and D. Yao, "A survey of traffic control with vehicular communications," IEEE Trans. Intell. Transp. Syst., vol. 15, no. 1, pp. 425-432, Feb. 2014.

[15] I. H. Zohdy and H. A. Rakha, "Intersection management via vehicle connectivity: The intersection cooperative adaptive cruise control system concept," J. Intell. Transp. Syst. Technol. Planning Oper, vol. 20, no. 1, pp. 17-32, 2016.

[16] B. Liu and A. El Kamel, "V2X-based decentralized cooperative adaptive cruise control in the vicinity of intersections," IEEE Trans. Intell. Transp. Syst., vol. 17, no. 3, pp. 644-658, Mar. 2016.

[17] M. Ahmane et al., "Modeling and controlling an isolated urban intersection based on cooperative vehicles," Transp. Res. C, Emerg. Technol., vol. 28, pp. 44-62, Mar. 2013.

[18] L. Li and F.-Y. Wang, "Cooperative driving at blind crossings using intervehicle communication," IEEE Trans. Veh. Technol., vol. 55, no. 6 , pp. 1712-1724, Nov. 2006.

[19] R. Sengupta, S. Rezaei, S. E. Shladover, D. Cody, S. Dickey, and H. Krishnan, "Cooperative collision warning systems: Concept definition and experimental implementation," J. Intell. Transp. Syst. Technol. Planning Oper., vol. 11, no. 3, pp. 143-155, 2007.

[20] J.-A. Jang, K. Choi, and H. Cho, "A fixed sensor-based intersection collision warning system in vulnerable line-of-sight and/or traffic-violationprone environment," IEEE Trans. Intell. Transp. Syst., vol. 13, no. 4, pp. 1880-1890, Dec. 2012.

[21] Cooperative Intersection Collision Avoidance Systems (CICAS). Accessed on Apr. 16, 2016. [Online]. Available: http:/its.dot.gov/ cicas/index.htm

[22] G. S. Aoude, V. R. Desaraju, L. H. Stephens, and J. P. How, "Driver behavior classification at intersections and validation on large naturalistic data set," IEEE Trans. Intell. Transp. Syst., vol. 13, no. 2, pp. 724-736, Jun. 2012 
[23] E. Ward and J. Folkesson, "Multi-classification of driver intentions in yielding scenarios," in Proc. IEEE Conf. Intell. Transp. Syst. (ITSC), Sep. 2015, pp. 678-685.

[24] B. Roessler and K. Fuerstenberg, "First european STREP on cooperative intersection safety, INTERSAFE-2," presented at the IEEE Conf. Intell. Transp. Syst. (ITSC), Sep. 2010, pp. 422-427.

[25] O. Aycard et al., "Intersection safety using lidar and stereo vision sensors," presented at the IEEE Intell. Vehicles Symp., Jun. 2011, pp. 863-869.

[26] F. Biral, M. Da Lio, R. Lot, and R. Sartori, "An intelligent curve warning system for powered two wheel vehicles," Eur. Transp. Res. Rev., vol. 2, no. 3, pp. 147-156, 2010.

[27] F. Biral, R. Lot, S. Rota, M. Fontana, and V. Huth, "Intersection support system for powered two-wheeled vehicles: Threat assessment based on a receding horizon approach," IEEE Trans. Intell. Transp. Syst., vol. 13, no. 2, pp. 805-816, Jun. 2012

[28] T. J. Prescott, P. Redgrave, and K. Gurney, "Layered control architectures in robots and vertebrates," Adapt. Behav., vol. 7, no. 1, pp. 99-127, Jan. 1999.

[29] R. Brooks, "A robust layered control system for a mobile robot," IEEE J. Robot. Autom., vol. RA-2, no. 1, pp. 14-23, Mar. 1986.

[30] P. Cisek, "Cortical mechanisms of action selection: The affordance competition hypothesis," Philos. Trans. Roy. Soc. London B, Biol. Sci., vol. 362, no. 1485, pp. 1585-1599, Sep. 2007.

[31] T. Flash and B. Hochner, "Motor primitives in vertebrates and invertebrates," Current Opinion Neurobiol., vol. 15, no. 6, pp. 660-666, Dec. 2005.

[32] F. A. Mussa-Ivaldi and S. A. Solla, "Neural primitives for motion control," IEEE J. Ocean. Eng., vol. 29, no. 3, pp. 640-650, Jul. 2004.

[33] D. Windridge, A. Shaukat, and E. Hollnagel, "Characterizing driver intention via hierarchical perception-action modeling," IEEE Trans. Human-Mach. Syst., vol. 43, no. 1, pp. 17-31, Jan. 2013.

[34] E. Todorov and M. I. Jordan, "Optimal feedback control as a theory of motor coordination," Nature Neurosci., vol. 5, no. 11, pp. 1226-1235, 2002.

[35] E. Todorov, "Optimality principles in sensorimotor control (review)," Nature Neurosci., vol. 7, no. 9, pp. 907-915, Sep. 2004.

[36] D. Liu and E. Todorov, "Evidence for the flexible sensorimotor strategies predicted by optimal feedback control," J. Neurosci., vol. 27, no. 35 , pp. 9354-9368, Aug. 2007.

[37] A. Simpkins and E. Todorov, "Complex object manipulation with hierarchical optimal control," in Proc. IEEE Symp. Adapt. Dyn. Program. Reinforcement Learn. (ADPRL), Apr. 2011, pp. 338-345.

[38] A. J. Nagengast, D. A. Braun, and D. M. Wolpert, "Optimal control predicts human performance on objects with internal degrees of freedom," PLoS Comput. Biol., vol. 5, no. 6, p. e1000419, Jun. 2009.

[39] P. M. Bays and D. M. Wolpert, "Computational principles of sensorimotor control that minimize uncertainty and variability," J. Physiol., vol. 578, no. 2, pp. 387-396, Jan. 2007.

[40] C. M. Harris and D. M. Wolpert, "Signal-dependent noise determines motor planning," Nature, vol. 394, pp. 780-784, Aug. 1998.

[41] C. M. Harris, "Biomimetics of human movement: Functional or aesthetic?" Bioinspiration Biomimetics, vol. 4, no. 3, p. 33001, Sep. 2009.

[42] P. Viviani and T. Flash, "Minimum-jerk, two-thirds power law, and isochrony: Converging approaches to movement planning," J. Experim. Psychol. Hum. Perception Perform., vol. 21, no. 1, pp. 32-53, Mar. 1995.

[43] H. J. Amin and A. K. Maurya, "A review of critical gap estimation approaches at uncontrolled intersection in case of heterogeneous traffic conditions," J. Transp. Literature, vol. 9, no. 3, pp. 5-9, Jul./Sep. 2015.

[44] P. Redgrave, T. J. Prescott, and K. Gurney, "The basal ganglia: A vertebrate solution to the selection problem?" Neuroscience, vol. 89, pp. 1009-1023, Apr. 1999.

[45] R. Bogacz and K. Gurney, "The basal ganglia and cortex implement optimal decision making between alternative actions," Neural Comput., vol. 19, no. 2, pp. 442-477, 2007.

[46] K. Doya, "What are the computations of the cerebellum, the basal ganglia and the cerebral cortex?" Neural Netw., vol. 12, nos. 7-8, pp. 961-974, Oct. 1999.

[47] P. Bosetti, M. Da Lio, and A. Saroldi, "On the human control of vehicles: An experimental study of acceleration," Eur. Transp. Res. Rev., vol. 6, no. 2, pp. 157-170, 2014

[48] P. Bosetti, M. Da Lio, and A. Saroldi, "On curve negotiation: From driver support to automation," IEEE Trans. Intell. Transp. Syst., vol. 16, no. 4, pp. 2082-2093, Aug. 2015.
[49] M. Da Lio, A. Mazzalai, K. Gurney, and A. Saroldi, "Biologically guided driver modeling: The stop behavior of human car drivers," IEEE Trans. Intell. Transp. Syst., to be published.

[50] D. Ruscio, M. R. Ciceri, and F. Biassoni, "How does a collision warning system shape driver's brake response time? The influence of expectancy and automation complacency on real-life emergency braking," Accident Anal. Prevention, vol. 77, pp. 72-81, Apr. 2015.

[51] F. Biral, D. Bortoluzzi, V. Cossalter, and M. Da Lio, "Experimental study of motorcycle transfer functions for evaluating handling," Vehicle Syst. Dyn., vol. 39, no. 1, pp. 1-25, 2003.

[52] J. R. Mclean and E. R. Hoffmann, "Analysis of driver's control movements," Hum. Factors, J. Hum. Factors Ergonom. Soc., vol. 13, no. 5, pp. 407-418, Oct. 1971.

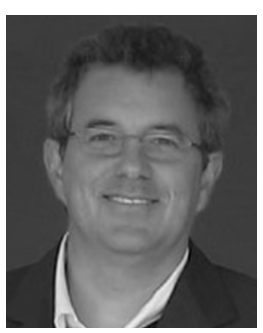

Mauro Da Lio received the Laurea degree in mechanical engineering from the University of Padova, Italy, in 1986. He is currently a Full professor of mechanical systems with the University of Trento, Italy. His earlier research activity was on modeling, simulation and optimal control of mechanical multibody systems, in particular vehicle, and spacecraft dynamics. More recently his focus shifted to the modeling of human sensory-motor control, in particular drivers and motor impaired people. Prior to his academic career, he was involved in offshore oil research company in underwater robotics a EUREKA Project. He was involved in several EU Framework Program 6 and 7 Projects (PReVENT, SAFERIDER, interactIVe, VERITAS, AdaptIVe, and NoTremor). $\mathrm{He}$ is currently the Coordinator of the EU Horizon 2020 Dreams4Cars Research and Innovation Action: a collaborative project in the Robotics domain which aims at increasing the cognition abilities of artificial driving agents by means of offline simulation mechanisms broadly inspired to the human dream state.

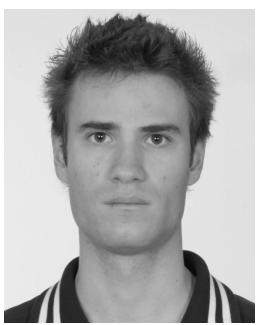

Alessandro Mazzalai received the B.S. degree in industrial engineering and the M.Sc. degree in mechatronics engineering from the University of Trento, Italy in 2011 and 2013, respectively, where he is currently pursuing the Ph.D. degree in mechatronic engineering. Since 2014, he has been involved in the European FP7 Project AdaptIVe and since the same year he has been Teaching Assistant with the Department of Industrial Engineering of the University of Trento. His research interests include automated driving in urban scenarios, the development of an artificial driver, and the study of vehicle dynamics.

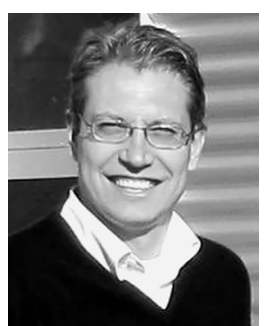

Marco Darin received the Laurea degree in computer science engineering from the University of Padova, Italy, in 2001. Since 2001 he has been with the Centro Ricerche Fiat, Microsystems Unit, as Designer and Developer of in-vehicle embedded systems. Since 2006, he is the Head of the InfoTelematic Systems unit coordinating all the activities related to FCA Research And Development Programs for vehicle connectivity, positioning and smart applications. In particular, to provide distinctiveness on V2X applications, to support emerging cooperative systems and autonomous driving solutions. He was involved in a number of EU and National Projects on microsystems, intelligent mobility, preventive safety and vehicular communication FeedMAP, Safespot, DRIVEC2X, MOTO, OpenGate, SIRPA, HF-ProtoCell, SOLCO, and e2Call. 